\title{
A Report on COVID-19 Epidemic in Pakistan: An SEIR Fractional Model
}

\section{Zubair Ahmad}

Department of Mathematics, City University of Science and Information Technology, Peshawar 25000, Khyber Pakhtunkhwa, Pakistan.

\section{Muhammad Arif}

Department of Mathematics, City University of Science and Information Technology, Peshawar 25000, Khyber Pakhtunkhwa, Pakistan.

\section{Farhad Ali ( $\square$ farhad.ali@tdtu.edu.vn )}

Computational Analysis Research Group, Ton Duc Thang University, Ho Chi Minh City 70000, Vietnam. 3Faculty of Mathematics and Statistics, Ton Duc Thang University, Ho Chi Minh City 70000, Vietnam llyas Khan

Department of Mathematics, College of Science Al-Zulfi, Majmaah University, Al-Majmaah 11952, Saudi Arabia.

\section{Kottakkaran Sooppy}

Department of Mathematics, College of Arts and Science, Wadi Al-Dawaser, 11991, Prince Sattam bin Abdulaziz University, Saudi Arabia

\section{Research Article}

Keywords: COVID-19, SEIR model, AB fractional model, Stability analysis, real data, numerical results

Posted Date: June 30th, 2020

DOl: https://doi.org/10.21203/rs.3.rs-37630/v1

License: (9) This work is licensed under a Creative Commons Attribution 4.0 International License. Read Full License 


\title{
A Report on COVID-19 Epidemic in Pakistan: An SEIR Fractional Model
}

\author{
Zubair Ahmad
Nisar $^{5}$, Muhammad Arif \\ ${ }^{1}$ Department of Mathematics, City University of Science and Information Technology, Peshawar 25000, Khyber \\ Pakhtunkhwa, Pakistan. \\ ${ }^{2}$ Computational Analysis Research Group, Ton Duc Thang University, Ho Chi Minh City 70000, Vietnam. \\ ${ }^{3}$ Faculty of Mathematics and Statistics, Ton Duc Thang University, Ho Chi Minh City 70000, Vietnam. \\ ${ }^{4}$ Department of Mathematics, College of Science Al-Zulfi, Majmaah University, Al-Majmaah 11952, Saudi \\ Arabia. \\ ${ }_{5}^{5}$ Department of Mathematics, College of Arts and Science, Wadi Al-Dawaser, 11991, Prince Sattam bin Abdulaziz \\ University, Saudi Arabia \\ *Corresponding author: Farhad Ali (e-mail: farhad.ali@tdtu.edu.vn).
}

\section{Abstract:}

Recently, novel coronavirus is a serious global issue and having a negative impact on the economy of the whole world. Like other countries, it also effected the economy and people of Pakistan. According to the publicly reported data, the first case of novel corona virus in Pakistan was reported on $27^{\text {th }}$ February 2020. The aim of the present study is to describe the mathematical model and dynamics of COVID-19 in Pakistan. To investigate the spread of coronavirus in Pakistan, we develop the SEIR time fractional model with newly, developed fractional operator of Atangana-Baleanu. We present briefly the analysis of the given model and discuss its applications using world health organization (WHO) reported data for Pakistan. We consider the available infection cases from $19^{\text {th }}$ march 2020 , till $31^{\text {st }}$ march 2020 and accordingly, various parameters are fitted or estimated. It is worth noting that we have calculated the basic reproduction number $\mathfrak{R}_{0} \approx 2.30748$ which shows that virus is spreading rapidly. Furthermore, stability analysis of the model at disease free equilibrium DFE and endemic equilibriums EE is performed to observe the dynamics and transmission of the model. Finally, the AB fractional model is solved numerically. To show the effect of the various embedded parameters like fractional parameter $\alpha$ on the model, various graphs are plotted. It is worth noting that the base of our investigation, we have predicted the spread of disease for next 200 days.

Keywords: COVID-19; SEIR model; AB fractional model; Stability analysis; real data; numerical results. 


\section{Introduction:}

From the beginning the world is constantly facing disasters, in the form of earth quakes, tsunami, floods and pandemics occurred in different times and places. For example in different times the human being suffered by different kinds of infectious diseases, like HIV, Ebola virus, bird flu, dengue virus, malaria, TB, hepatitis B and C, diarrhea, influenza, chicken pox and rubella disease etc. These infectious diseases affected humans as well as other animals. Beside this, these infectious diseases not only spread in human and animals but it also spread in plants like, Pine wilt disease, Moko diseases, sugar cane, orange rust, Karnal bunt diseases and Dutchelm etc [1]. These pandemics not only affected the economy of the world but also caused many deaths of mankind. The study of these infectious diseases is very important to the investigate the spread of infectious diseases and to find out the behavior of these infectious diseases on living organisms. To look for the possible available resources which help to detect these diseases and prevent from them. The duty of humankind is to create a healthy environment in which they live. The growth in human population is increasing with a great speed which also effect the environment. The nature of spreading of these viruses are different, some of them are fast spreading viruses while others are slow in spreading. In-spite of the spreading nature of these infectious diseases, they affected many humans as well as plants.

As discussed above, viruses may have different nature and having some specific visible history for different stages of spreading these infectious diseases. In the present study we formulate the model for the globally spreading infectious disease namely novel corona virus (COVID-19). The first case of novel corona virus COVID-19 in human was reported in Wuhan China on December $31^{\text {st }}$ 2019. Initially, the symptoms of this virus was considered as pneumonia. After the vaccination and treatment of pneumonia the infected patient was not recovered and the treatment was not effective [2]. Secondly, it was observed that the transmission of this virus was very fast from human to human in China. Furthermore, the infected cases was not limited to the city of China Wuhan, it also spread in other cities of China [3]. As the presence of this virus was not limited to China, but also spread in different region of the world. It can be noticed from the behavior of corona virus that initially, it was considered as epidemic disease. But the cases of corona virus spread in the whole world and then it became pandemic disease. Furthermore, it was found that symptoms of corona virus in human being takes 2 to 10 days [4]. 
As this deadly virus spread rapidly in China and in the whole world, like Europe, North America, Germany and Itly and caused many deaths. The number of deaths due to COVID-19 was record in thousands. This virus also affect Asian countries specially Pakistan. In Pakistan the first case of Corona virus was detected in $27^{\text {th }}$ February 2020 [5]. From the $27^{\text {th }}$ February 2020 till to $31^{\text {st }}$ March 2020, the number of cases of COVID-19, increased day by day. According to the data collected by WHO [5] the number of total infected cases were 1865 and total reported deaths were 25 till $31^{\text {st }}$ March 2020.

The aim of present study is to investigate the dynamics and transmission of COVID-19 SEIR model. Furthermore, we transformed the classical model by newly developed AtanganaBaleanu time fractional model. The purpose of using $\mathrm{AB}$ time fractional derivative is that it has non-singular and non-local kernel and it may predict the spread COVID-19 accurately. Recently, many researchers have chosen $\mathrm{AB}$ fractional model for dynamics of different infectious diseases, like the dynamics of HIV [6], chicken pox [7], hepatitis E [8], dengue fever [9], rubella disease [10], measles [11], tuberculosis [12]. The applications of AB fractional derivative is not only limited to study the dynamics of infectious diseases but it has many applications in modern sciences and technologies e.g. nanofluids [13], biofluids [14], solar collectors [15], chaotic processes [16], electric circuits [17] etc.

More precisely, in the present research, we have taken the data of COVID-19 from $19^{\text {th }}$ march 202 to $31^{\text {st }}$ march 2020 and parameterized the given model to the real data. Positivity and boundedness of the present model is also discussed. Fixed points and basic reproduction number is estimated. Stability analysis and some basic properties have been proved for the given model. A numerical scheme is developed for the given fractional model and displayed its results for the fractional parameter $\alpha$ through graphs. Some figures are plotted with different initial conditions for global asymptotical stability (GAS).

\section{Mathematical Modeling:}

In the present mathematical model, we have considered the spread of COVID-19 in human. The total population is denoted by $N_{h}(\tau)$ which is further subdivided into four subclasses. The four subclasses are susceptible, exposed, infected and recovered people which are denoted by $S_{h}(\tau), E_{h}(\tau), I_{h}(\tau)$ and $R_{h}(\tau)$ respectively. The interaction among these subclasses has shown in the flow chart which is figure 1. 


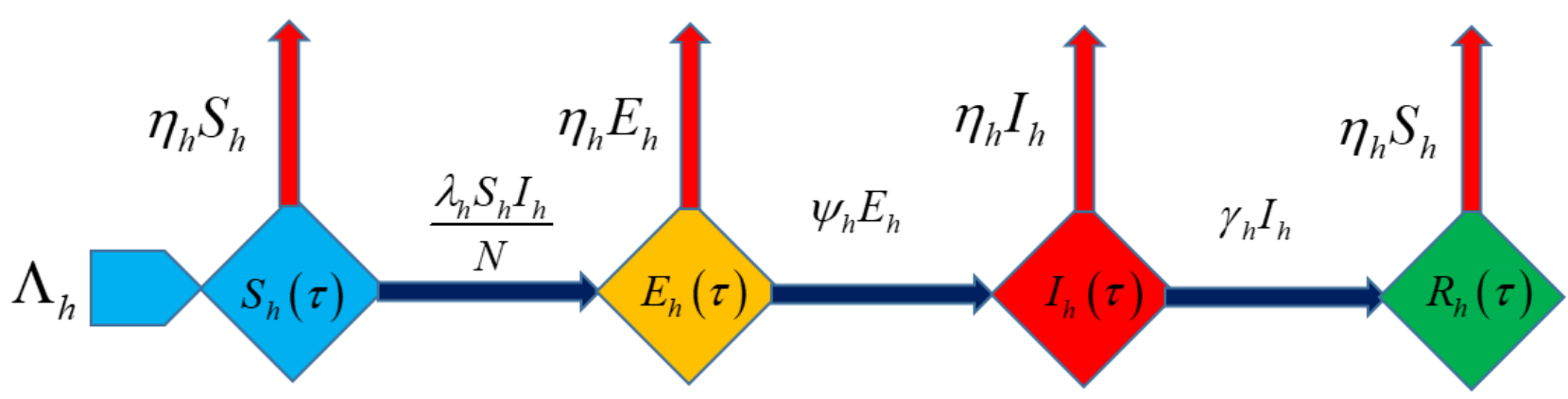

Figure 1. Flow chart of the given SEIR model.

In the given model, the recruitment rate of susceptible population is $\Lambda_{h}$ which represents birth rate, $\eta_{h}$ represents death rate of each subclass, $\lambda_{h}$ represents interaction rate between susceptible and infected population with the route $\frac{\lambda_{h} S_{h} I_{h}}{N}, \psi_{h}$ represents the rate at which exposed class completed their incubation period and enter into the infected class, $\gamma_{h}$ is the removal or recovery rate of infected population.

$$
\left.\begin{array}{l}
\frac{d S_{h}(\tau)}{d \tau}=\Lambda_{h}-\eta_{h} S_{h}-\frac{\lambda_{h} S_{h} I_{h}}{N}, \\
\frac{d E_{h}(\tau)}{d \tau}=\frac{\lambda_{h} S_{h} I_{h}}{N}-\eta_{h} E_{h}-\psi_{h} E_{h}, \\
\frac{d I_{h}(\tau)}{d \tau}=\psi_{h} E_{h}-\eta_{h} I_{h}-\gamma_{h} I_{h}, \\
\frac{d R_{h}(\tau)}{d \tau}=\gamma_{h} I_{h}-\eta_{h} R_{h},
\end{array}\right\}
$$

Corresponding non-negative initial conditions

$$
S(0)=S^{*} \geq 0, E(0)=E^{*} \geq 0, I(0)=I^{*} \geq 0 \text { and } R(0)=R^{*} \geq 0 .
$$

\section{Non-negativity and Boundedness of the Model:}

This section provided to prove the boundedness and positivity of the solutions of system (1). To show the positivity of the model (1), we state the following lemma:

Lemma 3.1. Suppose $\Phi \subset \mathbb{R} \times \mathbb{C}^{n}$ is open, $g_{i} \in \mathbb{C}(\Phi, \mathbb{R}), i=1,2,3, \ldots, n$. If $\left.g_{i}\right|_{x_{i}(\tau)=0, X_{\tau} \in \mathbb{C}_{+0}^{n}} \geq 0$ , $X_{\tau}=\left(x_{1 \tau}, x_{2 \tau}, \ldots, x_{n \tau}\right)^{T}, i=1,2, \ldots, n$, then $\mathbb{C}_{+0}^{n}\left\{\varphi=\left(\varphi_{1}, \varphi_{2}, \ldots, \varphi_{n}\right): \varphi \in \mathbb{C}\left([-v, 0], \mathbb{R}_{+0}^{n}\right)\right\}$ is the invariant domain of the following equations.

$$
\frac{d x_{i}(\tau)}{d \tau}=g_{i}\left(\tau, X_{\tau}\right), \tau \geq \sigma, i=1,2, \ldots, n .
$$


Where $\mathbb{R}_{+0}^{n}\left\{\left(x_{1}, x_{2}, \ldots, x_{n}\right): x_{i} \geq 0, i=1,2, \ldots, n\right\}$

Preposition 3.1. The system (1) is invariant in $\mathbb{R}_{+}^{4}$.

Proof: By rewriting the system (1), we have:

$$
\begin{aligned}
& \frac{d X}{d \tau}=M(X(\tau)), X(0)=X_{0} \geq 0, \\
& M(X(\tau))=\left(M_{1}(X), M_{2}(X), M_{3}(X), M_{4}(X)\right)^{T},
\end{aligned}
$$

We noted that

$$
\left.\begin{array}{l}
\left.\frac{d S_{h}(\tau)}{d \tau}\right|_{S_{h}=0}=\Lambda_{h} \geq 0 \\
\left.\frac{d E_{h}(\tau)}{d \tau}\right|_{E_{h}=0}=\frac{\lambda_{h} S_{h} I_{h}}{S_{h}+I_{h}+R_{h}} \geq 0 \\
\left.\frac{d I_{h}(\tau)}{d \tau}\right|_{I_{h}=0}=\psi_{h} E_{h} \geq 0 \\
\left.\frac{d R_{h}(\tau)}{d \tau}\right|_{R_{h}=0}=\gamma_{h} I_{h} \geq 0
\end{array}\right\},
$$

According to Lemma 3.1, $\mathbb{R}_{+}^{4}$ is invariant set.

Preposition 3.2. The system (1) is bounded in the region:

$$
\Phi=\left\{\left(S_{h}(\tau), E_{h}(\tau), I_{h}(\tau), R_{h}(\tau)\right) \in \mathbb{R}^{4}: N_{h}(\tau) \leq \frac{\Lambda_{h}}{\eta_{h}}\right\} .
$$

Proof: Boundedness of the problem (1) can be verified by adding all equations of system (1), we obtained:

$$
\frac{d N_{h}(\tau)}{d \tau}=\Lambda_{h}-\eta_{h} N_{h}, \quad \text { with } \quad N_{h}(0)=N_{0} \geq 0
$$

The solution of equation (8) takes the form:

$$
N_{h}(\tau) \leq N_{0} e^{-\eta_{h} \tau}+\frac{\Lambda_{h}}{\eta_{h}}\left(1-e^{-\eta_{h} \tau}\right)
$$

It can be clearly seen from equation (9) that if $\tau \rightarrow \infty$ then $N_{h}(\tau) \leq \frac{\Lambda_{h}}{\eta_{h}}$ which means that the feasible region for the given model will be: 


$$
\Phi=\left\{\left(S_{h}(\tau), E_{h}(\tau), I_{h}(\tau), R_{h}(\tau)\right) \in \mathbb{R}^{4}: N_{h}(\tau) \leq \frac{\Lambda_{h}}{\eta_{h}}\right\}
$$

Hence the solution of the system (1) is bounded.

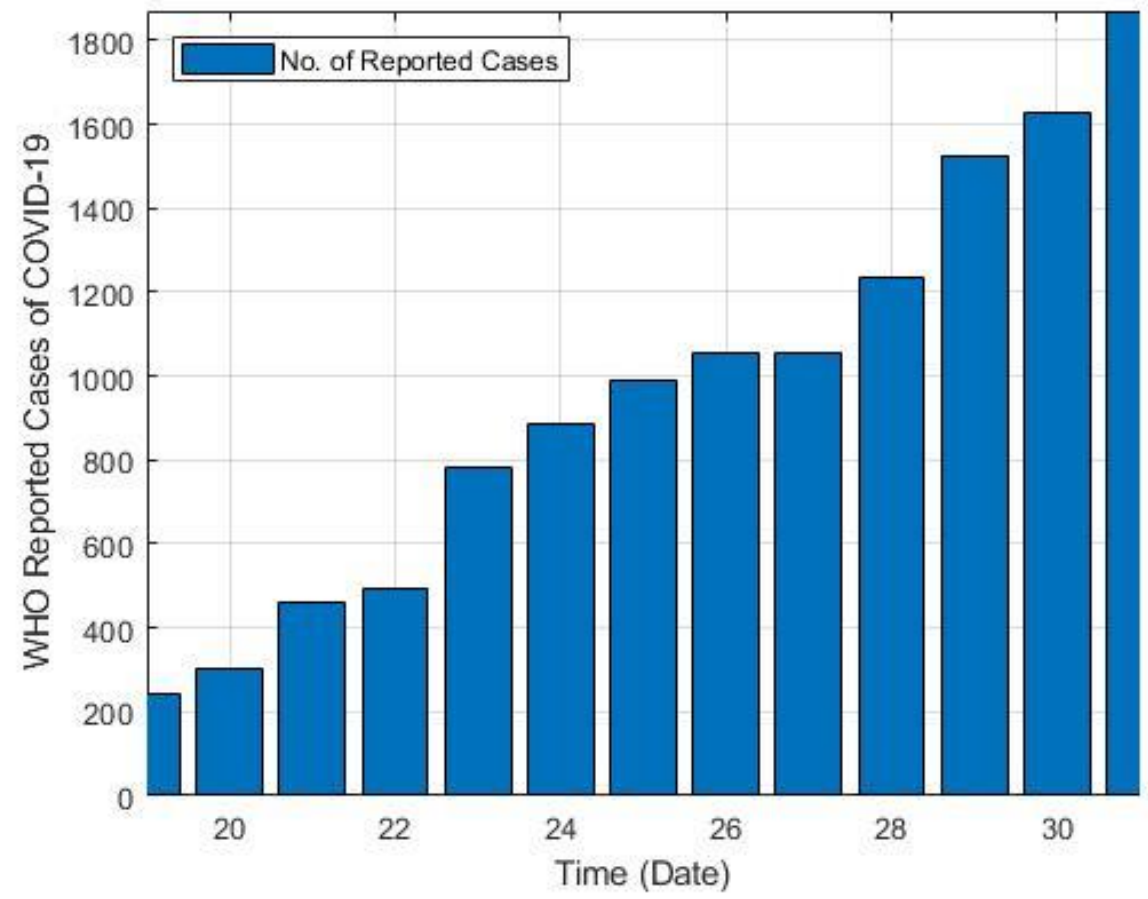

Figure 2. WHO reported cases for COVID-19 from $19^{\text {th }}$ March 2020 till $31^{\text {st }}$ March 2020.

\section{Equilibrium points, Basic reproduction number and local stability analysis}

This section includes the possible fixed points of model (1). There exists two possible equilibrium points are calculated, i.e. Disease free equilibrium (DFE) and endemic equilibrium (EE). Furthermore, basic reproduction number is calculated by next generation technique and discuss the local stable analysis of these equilibrium points. The steady state solution of model is given below by considering the rate of change w.r.t time becomes zero:

$$
\left.\frac{d S_{h}(\tau)}{d \tau}=\frac{d E_{h}(\tau)}{d \tau}=\frac{d I_{h}(\tau)}{d \tau}=\frac{d R_{h}(\tau)}{d \tau}=0,\right\}
$$

Using equation (11), model (1) becomes: 


$$
\left.\begin{array}{l}
0=\Lambda_{h}-\eta_{h} S_{h}-\frac{\lambda_{h} S_{h} I_{h}}{N}, \\
0=\frac{\lambda_{h} S_{h} I_{h}}{N}-\eta_{h} E_{h}-\psi_{h} E_{h}, \\
0=\psi_{h} E_{h}-\eta_{h} I_{h}-\gamma_{h} I_{h}, \\
0=\gamma_{h} I_{h}-\eta_{h} R_{h},
\end{array}\right\}
$$

From steady state system (12), DFE can be obtained by assuming $E_{h}=I_{h}=R_{h}=0$ and is denoted by

$$
\Psi_{D F E}=\left(S^{0}, E^{0}, I^{0}, R^{0}\right)=\left(\frac{\Lambda_{h}}{\eta_{h}}, 0,0,0\right)
$$

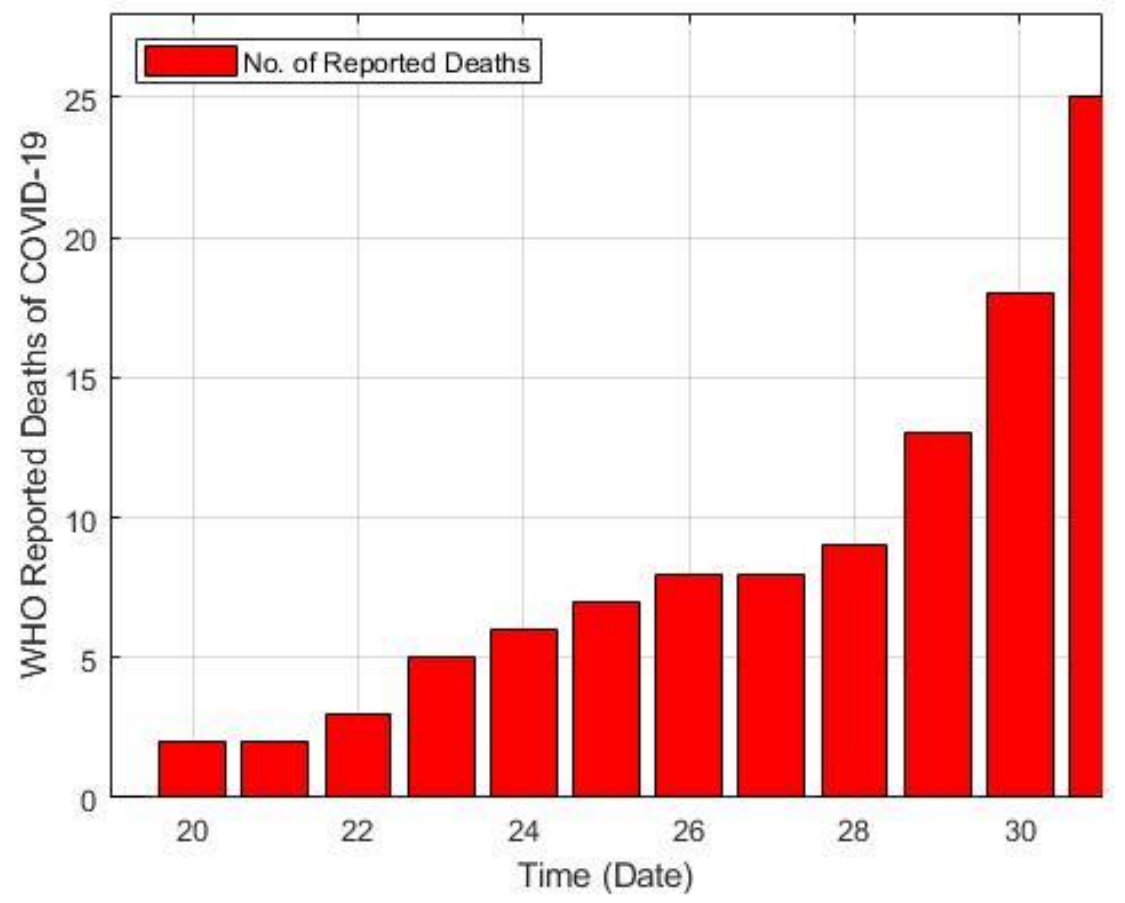

Figure 3. WHO reported deaths by COVID-19 from $19^{\text {th }}$ March 2020 till $31^{\text {st }}$ March 2020.

Similarly, EE of model (1) is obtained from system (12) and is given by:

$$
\Psi_{E E}=\left(S^{*}, E^{*}, I^{*}, R^{*}\right) \text {. }
$$

Where, 


$$
\left.\begin{array}{l}
S^{*}=\frac{\Lambda_{h}\left(\eta_{h}^{2}-\gamma_{h} \psi_{h}-\eta_{h} \psi_{h}\right)}{\eta_{h}\left(\eta_{h}^{2}+\eta_{h} \gamma_{h}+\eta_{h} \lambda_{h}-\eta_{h} \psi_{h}-\lambda_{h} \psi_{h}\right)}, \\
E^{*}=\frac{\Lambda_{h}\left(\eta_{h} \gamma_{h}+\eta_{h} \lambda_{h}+\gamma_{h} \psi_{h}-\lambda_{h} \psi_{h}\right)}{\left(\eta_{h}+\psi_{h}\right)\left(\eta_{h}^{2}+\eta_{h} \gamma_{h}+\eta_{h} \lambda_{h}-\eta_{h} \psi_{h}-\lambda_{h} \psi_{h}\right)}, \\
I^{*}=\frac{\Lambda_{h}\left(\psi_{h}-\eta_{h}\right)\left(\eta_{h} \gamma_{h}+\eta_{h} \lambda_{h}+\gamma_{h} \psi_{h}-\lambda_{h} \psi_{h}\right)}{\gamma_{h}\left(\eta_{h}+\psi_{h}\right)\left(\eta_{h}^{2}+\eta_{h} \gamma_{h}+\eta_{h} \lambda_{h}-\eta_{h} \psi_{h}-\lambda_{h} \psi_{h}\right)}, \\
R^{*}=\frac{\Lambda_{h}\left(\psi_{h}-\eta_{h}\right)\left(\eta_{h} \gamma_{h}+\eta_{h} \lambda_{h}+\gamma_{h} \psi_{h}-\lambda_{h} \psi_{h}\right)}{\eta_{h}\left(\eta_{h}+\psi_{h}\right)\left(\eta_{h}^{2}+\eta_{h} \gamma_{h}+\eta_{h} \lambda_{h}-\eta_{h} \psi_{h}-\lambda_{h} \psi_{h}\right)},
\end{array}\right\}
$$

The basic reproduction number $\mathfrak{R}_{0}$ is calculated by next generation technique [18]. The $F$ and $V$ matrices at DFE $\Psi^{0}$ is given as follows:

$$
\begin{aligned}
& F=\left[\begin{array}{cc}
0 & \lambda_{h} \\
0 & 0
\end{array}\right] \\
& V=\left[\begin{array}{cc}
\eta_{h}+\psi_{h} & 0_{h} \\
\psi_{h} & \eta_{h}+\gamma_{h}
\end{array}\right]
\end{aligned}
$$

According to [10] the spectral radius $\rho\left(F V^{-1}\right)$ is the required reproduction number $\mathfrak{R}_{0}$ which is calculated in the form:

$$
\mathfrak{R}_{0}=\frac{\lambda_{h} \psi_{h}}{\left(\eta_{h}+\psi_{h}\right)\left(\eta_{h}+\gamma_{h}\right)}
$$

Theorem 5.1. The DFE $\Psi^{0}$ of the system (1) is locally asymptotically stable if $\Re_{0}<1$.

Proof: The Jacobian matrix of system (1) at DFE is given by:

$$
J_{\Psi^{0}}=\left[\begin{array}{cccc}
-\eta_{h} & 0 & -\lambda_{h} & 0 \\
0 & -\eta_{h}-\psi_{h} & \lambda & 0 \\
0 & \psi_{h} & -\eta_{h}-\gamma_{h} & 0 \\
0 & 0 & \gamma_{h} & -\eta_{h}
\end{array}\right]
$$

Suppose $\lambda$ denote the eigen values of the Jacobian matrix $J_{\psi^{0}}$. Here, the two eigen values of the above matrix are negative i.e. $-\eta_{h}$ (twice). The remaining required eigen values can be obtained by the following characteristic equation:

$$
\lambda^{2}+\kappa_{1} \lambda+\kappa_{2}=0
$$

where 


$$
\begin{aligned}
& \kappa_{1}=\gamma_{h}+2 \eta_{h}+\psi_{h} \\
& \kappa_{2}=\eta_{h} \gamma+\eta_{h}^{2}+\gamma_{h} \psi_{h}-\lambda_{h} \psi_{h} \\
& \Rightarrow \kappa_{2}=\left(\eta_{h}+\gamma_{h}\right)\left(\eta_{h}+\psi_{h}\right)\left\{1-\mathfrak{R}_{0}\right\}
\end{aligned}
$$

From equation (17), it can be clearly noticed that $\kappa_{1}>0$. Similarly, from equation (18), it can be noticed that $\kappa_{2}>0$ when $\mathfrak{R}_{0}<1$. So, all the coefficients of characteristics equation are nonnegative. Furthermore, according to Rough-Hurtwiz criteria, the eigen values of the above chacteristics equation are negative. Thus, all the eigen values of Jacobian matrix (15) are negative for $\mathfrak{R}_{0}<1$. Hence the model (1) is locally asymptotically stable when $\mathfrak{R}_{0}<1$.

\section{Fractional model and Numerical Scheme}

\subsection{Preliminaries:}

Definition: The Atangana-Baleanu (AB) time fractional derivative with fractional order $\alpha$ is defined as [19].

$$
{ }_{a}^{A B} \wp_{\tau}^{\alpha} f(t)=\frac{\mathfrak{I}(\alpha)}{1-\alpha} \int_{a}^{\tau} E_{a}\left(\frac{-\alpha(\tau-t)^{\alpha}}{1-\alpha}\right) f^{\prime}(\tau) d t \text {, for } 0<\alpha<1 .
$$

Here, $\mathfrak{J}(\alpha)$ is the normalization function and $E_{\alpha}($.$) is Mittage-Leffler function [20].$

Definition: The numerical scheme for the solution of fractional order ODE is defined by Toufik and Atangana [21]:

Consider a non-linear fractional ODE:

$$
{ }^{A B} \wp_{\tau}^{\alpha} y(t)=f(t, y(t)) \text { with } y(0)=y_{0}
$$

The numerical scheme for equarion (23) is defined as [21]: 


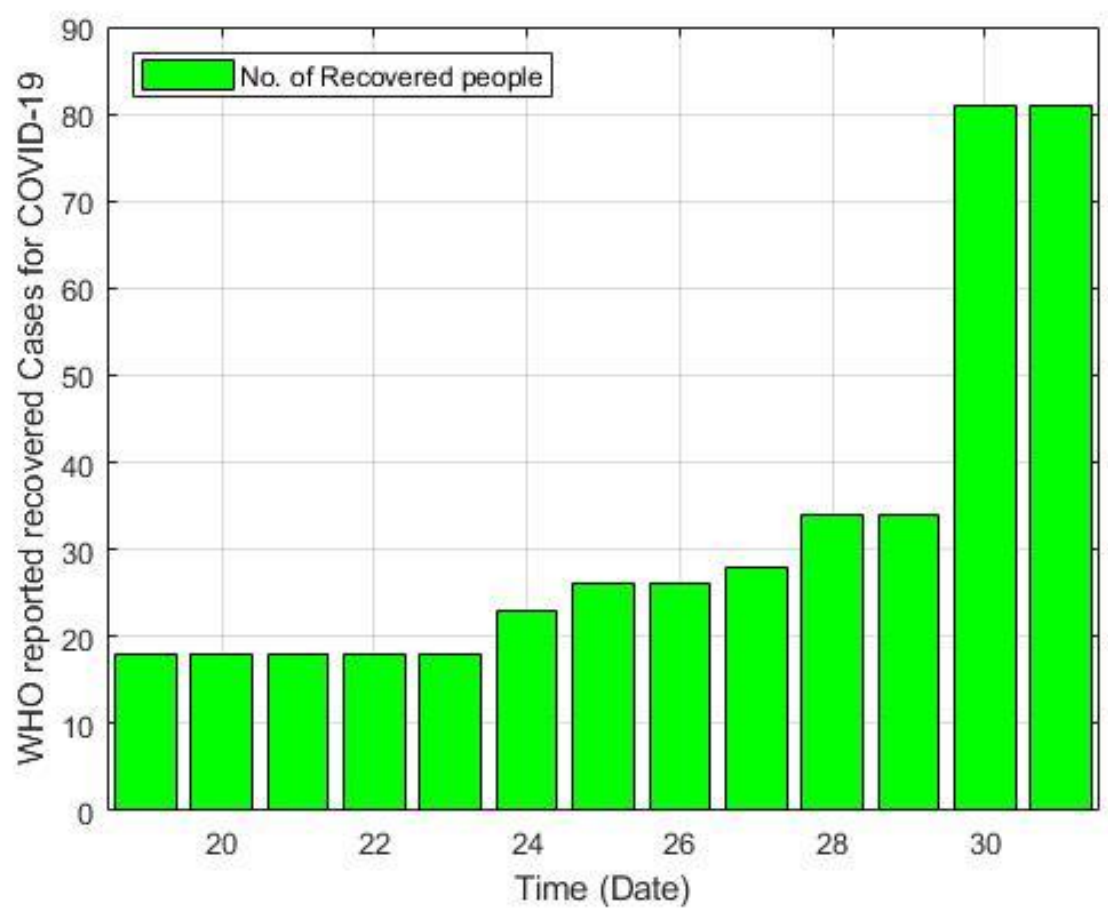

Figure 4. WHO reported deaths by COVID-19 from $19^{\text {th }}$ March 2020 till $31^{\text {st }}$ March 2020.

$$
\begin{aligned}
& y_{n+1}=y_{0}+\frac{1-\alpha}{\mathfrak{I}(\alpha)} f\left(t_{n}, y\left(t_{n}\right)\right) \\
& +\frac{\alpha}{\mathfrak{I}(\alpha)} \sum_{k=0}^{n}\left[\begin{array}{l}
\left.\frac{h^{\alpha} f\left(t_{k}, y\left(t_{k}\right)\right)}{\Gamma(\alpha+2)}\left\{(n+1-k)^{\alpha}(n+2-k+\alpha)-(n-k)^{\alpha}(n+2-k+2 \alpha)\right\}\right] \\
-\frac{h^{\alpha} f\left(t_{k-1}, y\left(t_{k-1}\right)\right)}{\Gamma(\alpha+2)}\left\{(n+1-k)^{\alpha+1}-(n-k)^{\alpha}(n+1-k+\alpha)\right\}
\end{array}\right]
\end{aligned}
$$

\subsection{Fractional model:}

For the generalization of model (1), we replace classical time derivative with AtanganaBaleanu time fractional derivative, model (1) takes the following generalized form:

$$
\left.\begin{array}{l}
{ }_{0}^{A B} \wp_{\tau}^{\alpha} S_{h}(\tau)=\Lambda_{h}-\eta_{h} S_{h}-\frac{\lambda_{h} S_{h} I_{h}}{N}, \\
{ }_{0}^{A B} \wp_{\tau}^{\alpha} E_{h}(\tau)=\frac{\lambda_{h} S_{h} I_{h}}{N}-\eta_{h} E_{h}-\psi_{h} E_{h}, \\
{ }_{0}^{A B} \wp_{\tau}^{\alpha} I_{h}(\tau)=\psi_{h} E_{h}-\eta_{h} I_{h}-\gamma_{h} I_{h}, \\
{ }_{0}^{A B} \wp_{\tau}^{\alpha} R_{h}(\tau)=\gamma_{h} I_{h}-\eta_{h} R_{h},
\end{array}\right\}
$$

Here, $\alpha$ is fractional parameter and ${ }_{0}^{A B} \wp_{\tau}^{\alpha}($.$) is the \mathrm{AB}$ time fractional derivative. All these variables and initial conditions for model (22) are positive. 


\subsection{Numerical Scheme:}

Adopting the procedure in [21], model (22) takes the following appropriate form:

$$
\left.\begin{array}{l}
{ }_{0}^{A B} \wp_{\tau}^{\alpha} S_{h}(\tau)=\chi_{1}\left(t, S_{h}, E_{h}, I_{h}, R_{h}\right), \\
{ }_{0}^{A B} \wp_{\tau}^{\alpha} E_{h}(\tau)=\chi_{2}\left(t, S_{h}, E_{h}, I_{h}, R_{h}\right), \\
{ }_{0}^{A B} \wp_{\tau}^{\alpha} I_{h}(\tau)=\chi_{3}\left(t, S_{h}, E_{h}, I_{h}, R_{h}\right), \\
{ }_{0}^{A B} \wp_{\tau}^{\alpha} R_{h}(\tau)=\chi_{4}\left(t, S_{h}, E_{h}, I_{h}, R_{h}\right),
\end{array}\right\}
$$

Furthermore, system (23) becomes:

$$
\begin{gathered}
S_{h}(\tau)-S_{h}(0)=\frac{1-\alpha}{\mathfrak{T}(\alpha)} \chi_{1}\left(\tau, S_{h}, E_{h}, I_{h}, R_{h}\right)+\frac{\alpha}{\mathfrak{I}(\alpha) \Gamma(\alpha)} \int_{0}^{\tau} \chi_{1}\left(\zeta, S_{h}, E_{h}, I_{h}, R_{h}\right)(\tau-\zeta)^{\alpha-1} d \zeta,(27) \\
E_{h}(\tau)-E_{h}(0)=\frac{1-\alpha}{\mathfrak{I}(\alpha)} \chi_{2}\left(\tau, S_{h}, E_{h}, I_{h}, R_{h}\right)+\frac{\alpha}{\mathfrak{I}(\alpha) \Gamma(\alpha)} \int_{0}^{\tau} \chi_{2}\left(\zeta, S_{h}, E_{h}, I_{h}, R_{h}\right)(\tau-\zeta)^{\alpha-1} d \zeta,(28) \\
I_{h}(\tau)-I_{h}(0)=\frac{1-\alpha}{\mathfrak{I}(\alpha)} \chi_{3}\left(\tau, S_{h}, E_{h}, I_{h}, R_{h}\right)+\frac{\alpha}{\mathfrak{I}(\alpha) \Gamma(\alpha)} \int_{0}^{\tau} \chi_{3}\left(\zeta, S_{h}, E_{h}, I_{h}, R_{h}\right)(\tau-\zeta)^{\alpha-1} d \zeta,(29) \\
R_{h}(\tau)-R_{h}(0)=\frac{1-\alpha}{\mathfrak{I}(\alpha)} \chi_{4}\left(\tau, S_{h}, E_{h}, I_{h}, R_{h}\right)+\frac{\alpha}{\mathfrak{I}(\alpha) \Gamma(\alpha)} \int_{0}^{\tau} \chi_{4}\left(\zeta, S_{h}, E_{h}, I_{h}, R_{h}\right)(\tau-\zeta)^{\alpha-1} d \zeta,(30)
\end{gathered}
$$

$$
+\frac{\alpha}{\Im}(\alpha) \Gamma(\alpha) \sum_{m=0}^{n} \int_{\tau_{m}}^{\tau_{m+1}} \chi_{3}\left(\zeta, S_{h}, E_{h}, I_{h}, R_{h}\right)\left(\tau_{n+1}-\zeta\right)^{\alpha-1} d \zeta,
$$




$$
\begin{aligned}
R\left(\tau_{n+1}\right)-R\left(\tau_{0}\right) & =\frac{1-\alpha}{\mathfrak{I}(\alpha)} \chi_{4}\left(\tau_{n}, S_{h}, E_{h}, I_{h}, R_{h}\right) \\
& +\frac{\alpha}{\mathfrak{I}(\alpha) \Gamma(\alpha)} \sum_{m=0}^{n} \int_{\tau_{m}}^{\tau_{m+1}} \chi_{4}\left(\zeta, S_{h}, E_{h}, I_{h}, R_{h}\right)\left(\tau_{n+1}-\zeta\right)^{\alpha-1} d \zeta
\end{aligned}
$$

Assuming the interval $\left[\tau_{m}, \tau_{m+1}\right]$ and using the two-step Lagrange polynomial interpolation, equations (31)-(34) becomes:

$$
\begin{aligned}
& S\left(\tau_{n+1}\right)-S\left(\tau_{0}\right)=\frac{1-\alpha}{\mathfrak{I}(\alpha)} \chi_{1}\left(\tau_{n}, S_{h}, E_{h}, I_{h}, R_{h}\right) \\
& +\frac{\alpha}{\Im}(\alpha) \Gamma(\alpha) \sum_{m=0}^{n}\left(\begin{array}{l}
\frac{\chi_{1}\left(\tau_{m}, S_{h}, E_{h}, I_{h}, R_{h}\right)}{l} \int_{\tau_{m}}^{\tau_{m+1}}\left(\zeta-\tau_{m+1}\right)^{\alpha-1}\left(\tau_{n+1}-\zeta\right)^{\alpha-1} d \zeta \\
-\frac{\chi_{1}\left(\tau_{m-1}, S_{h}, E_{h}, I_{h}, R_{h}\right)}{l} \int_{\tau_{m}}^{\tau_{m+1}}\left(\zeta-\tau_{m}\right)^{\alpha-1}\left(\tau_{n+1}-\zeta\right)^{\alpha-1} d \zeta
\end{array}\right) \\
& E\left(\tau_{n+1}\right)-E\left(\tau_{0}\right)=\frac{1-\alpha}{\mathfrak{I}(\alpha)} \chi_{2}\left(\tau_{n}, S_{h}, E_{h}, I_{h}, R_{h}\right) \\
& +\frac{\alpha}{\Im(\alpha) \Gamma(\alpha)} \sum_{m=0}^{n}\left(\begin{array}{c}
\frac{\chi_{2}\left(\tau_{m}, S_{h}, E_{h}, I_{h}, R_{h}\right)}{l} \int_{\tau_{m}}^{\tau_{m+1}}\left(\zeta-\tau_{m+1}\right)^{\alpha-1}\left(\tau_{n+1}-\zeta\right)^{\alpha-1} d \zeta \\
-\frac{\chi_{2}\left(\tau_{m-1}, S_{h}, E_{h}, I_{h}, R_{h}\right)}{l} \int_{\tau_{m}}^{\tau_{m+1}}\left(\zeta-\tau_{m}\right)^{\alpha-1}\left(\tau_{n+1}-\zeta\right)^{\alpha-1} d \zeta
\end{array}\right) \\
& I\left(\tau_{n+1}\right)-I\left(\tau_{0}\right)=\frac{1-\alpha}{\mathfrak{I}(\alpha)} \chi_{1}\left(\tau_{n}, S_{h}, E_{h}, I_{h}, R_{h}\right) \\
& +\frac{\alpha}{\mathfrak{I}(\alpha) \Gamma(\alpha)} \sum_{m=0}^{n}\left(\begin{array}{l}
\frac{\chi_{3}\left(\tau_{m}, S_{h}, E_{h}, I_{h}, R_{h}\right)^{\tau_{m+1}}}{l} \int_{\tau_{m}}^{\tau_{m}}\left(\zeta-\tau_{m+1}\right)^{\alpha-1}\left(\tau_{n+1}-\zeta\right)^{\alpha-1} d \zeta \\
-\frac{\chi_{3}\left(\tau_{m-1}, S_{h}, E_{h}, I_{h}, R_{h}\right)^{\tau_{m+1}}}{l} \int_{\tau_{m}}\left(\zeta-\tau_{m}\right)^{\alpha-1}\left(\tau_{n+1}-\zeta\right)^{\alpha-1} d \zeta
\end{array}\right) \\
& R\left(\tau_{n+1}\right)-R\left(\tau_{0}\right)=\frac{1-\alpha}{\mathfrak{I}(\alpha)} \chi_{1}\left(\tau_{n}, S_{h}, E_{h}, I_{h}, R_{h}\right) \\
& +\frac{\alpha}{\Im(\alpha) \Gamma(\alpha)} \sum_{m=0}^{n}\left(\begin{array}{c}
\frac{\chi_{4}\left(\tau_{m}, S_{h}, E_{h}, I_{h}, R_{h}\right)}{l} \int_{\tau_{m}}^{\tau_{m+1}}\left(\zeta-\tau_{m+1}\right)^{\alpha-1}\left(\tau_{n+1}-\zeta\right)^{\alpha-1} d \zeta \\
-\frac{\chi_{4}\left(\tau_{m-1}, S_{h}, E_{h}, I_{h}, R_{h}\right)}{l} \int_{\tau_{m}}^{\tau_{m+1}}\left(\zeta-\tau_{m}\right)^{\alpha-1}\left(\tau_{n+1}-\zeta\right)^{\alpha-1} d \zeta
\end{array}\right)
\end{aligned}
$$

Where $l$ is the step size.

By solving the integration terms in the above equations, equations (35)-(38) gets the following iterative shape: 


$$
\begin{aligned}
& S\left(\tau_{n+1}\right)=S\left(\tau_{0}\right)+\frac{1-\alpha}{\mathfrak{I}(\alpha)} \chi_{1}\left(t_{n}, S_{h}, E_{h}, I_{h}, R_{h}\right) \\
& +\frac{\alpha}{\mathfrak{I}(\alpha)} \sum_{m=0}^{n}\left[\begin{array}{l}
\left.\frac{l^{\alpha} \chi_{1}\left(t_{m}, S_{h}, E_{h}, I_{h}, R_{h}\right)}{\Gamma(\alpha+2)}\left\{(n+1-m)^{\alpha}(n+2-m+\alpha)-(n-m)^{\alpha}(n+2-m+2 \alpha)\right\}\right],(39) \\
-\frac{l^{\alpha} \chi_{1}\left(t_{m-1}, S_{h}, E_{h}, I_{h}, R_{h}\right)}{\Gamma(\alpha+2)}\left\{(n+1-m)^{\alpha+1}-(n-m)^{\alpha}(n+1-m+\alpha)\right\}
\end{array}\right. \\
& E\left(\tau_{n+1}\right)=E\left(\tau_{0}\right)+\frac{1-\alpha}{\mathfrak{I}(\alpha)} \chi_{2}\left(t_{n}, S_{h}, E_{h}, I_{h}, R_{h}\right) \\
& +\frac{\alpha}{\mathfrak{I}(\alpha)} \sum_{m=0}^{n}\left[\begin{array}{l}
\frac{l^{\alpha} \chi_{2}\left(t_{m}, S_{h}, E_{h}, I_{h}, R_{h}\right)}{\Gamma(\alpha+2)}\left\{(n+1-m)^{\alpha}(n+2-m+\alpha)-(n-m)^{\alpha}(n+2-m+2 \alpha)\right\} \\
-\frac{l^{\alpha} \chi_{2}\left(t_{m-1}, S_{h}, E_{h}, I_{h}, R_{h}\right)}{\Gamma(\alpha+2)}\left\{(n+1-m)^{\alpha+1}-(n-m)^{\alpha}(n+1-m+\alpha)\right\}
\end{array}\right]
\end{aligned}
$$
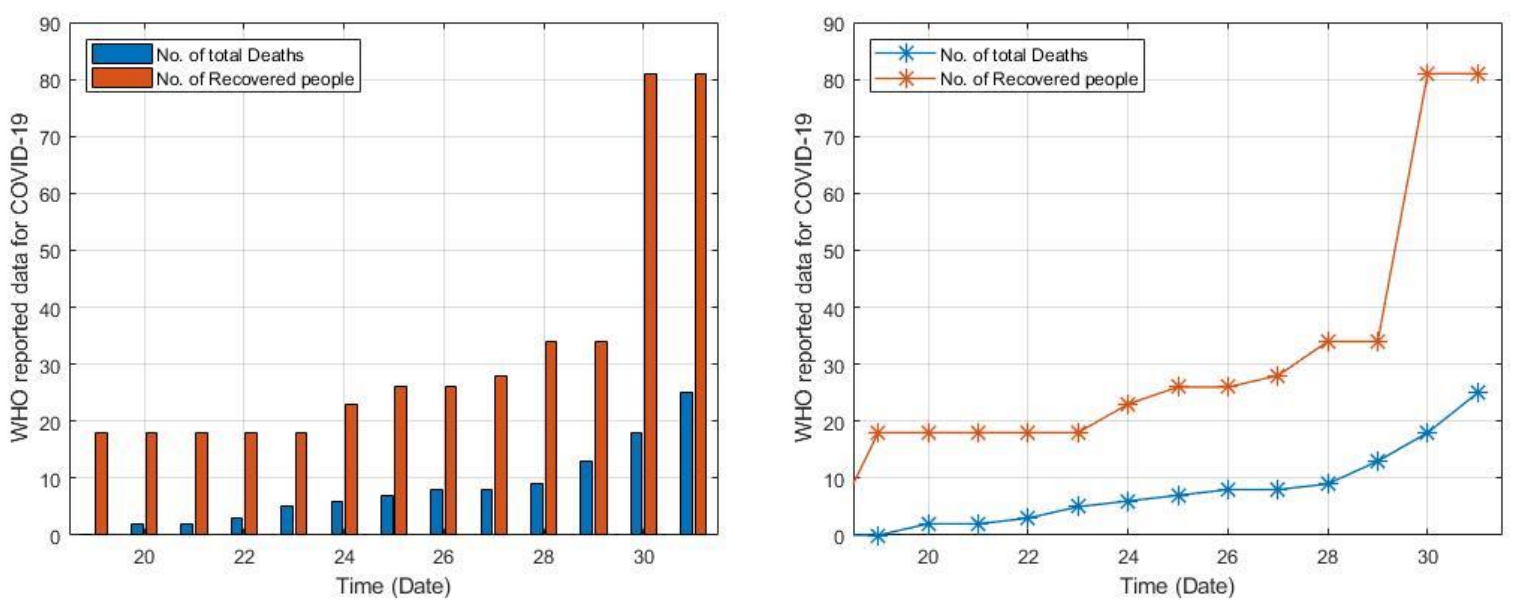

Figure 5. Comparative bar chart and line plot of reported deaths and recovered individuals of COVID-19

$$
\begin{aligned}
& I\left(\tau_{n+1}\right)=I\left(\tau_{0}\right)+\frac{1-\alpha}{\mathfrak{I}(\alpha)} \chi_{3}\left(t_{n}, S_{h}, E_{h}, I_{h}, R_{h}\right) \\
& +\frac{\alpha}{\mathfrak{I}(\alpha)} \sum_{m=0}^{n}\left[\begin{array}{l}
\frac{l^{\alpha} \chi_{3}\left(t_{m}, S_{h}, E_{h}, I_{h}, R_{h}\right)}{\Gamma(\alpha+2)}\left\{(n+1-m)^{\alpha}(n+2-m+\alpha)-(n-m)^{\alpha}(n+2-m+2 \alpha)\right\} \\
-\frac{l^{\alpha} \chi_{3}\left(t_{m-1}, S_{h}, E_{h}, I_{h}, R_{h}\right)}{\Gamma(\alpha+2)}\left\{(n+1-m)^{\alpha+1}-(n-m)^{\alpha}(n+1-m+\alpha)\right\}
\end{array}\right] \\
& R\left(\tau_{n+1}\right)=R\left(\tau_{0}\right)+\frac{1-\alpha}{\mathfrak{I}(\alpha)} \chi_{4}\left(t_{n}, S_{h}, E_{h}, I_{h}, R_{h}\right) \\
& +\frac{\alpha}{\mathfrak{I}(\alpha)} \sum_{m=0}^{n}\left[\begin{array}{l}
\frac{l^{\alpha} \chi_{4}\left(t_{m}, S_{h}, E_{h}, I_{h}, R_{h}\right)}{\Gamma(\alpha+2)}\left\{(n+1-m)^{\alpha}(n+2-m+\alpha)-(n-m)^{\alpha}(n+2-m+2 \alpha)\right\} \\
-\frac{l^{\alpha} \chi_{4}\left(t_{m-1}, S_{h}, E_{h}, I_{h}, R_{h}\right)}{\Gamma(\alpha+2)}\left\{(n+1-m)^{\alpha+1}-(n-m)^{\alpha}(n+1-m+\alpha)\right\}
\end{array}\right]
\end{aligned}
$$




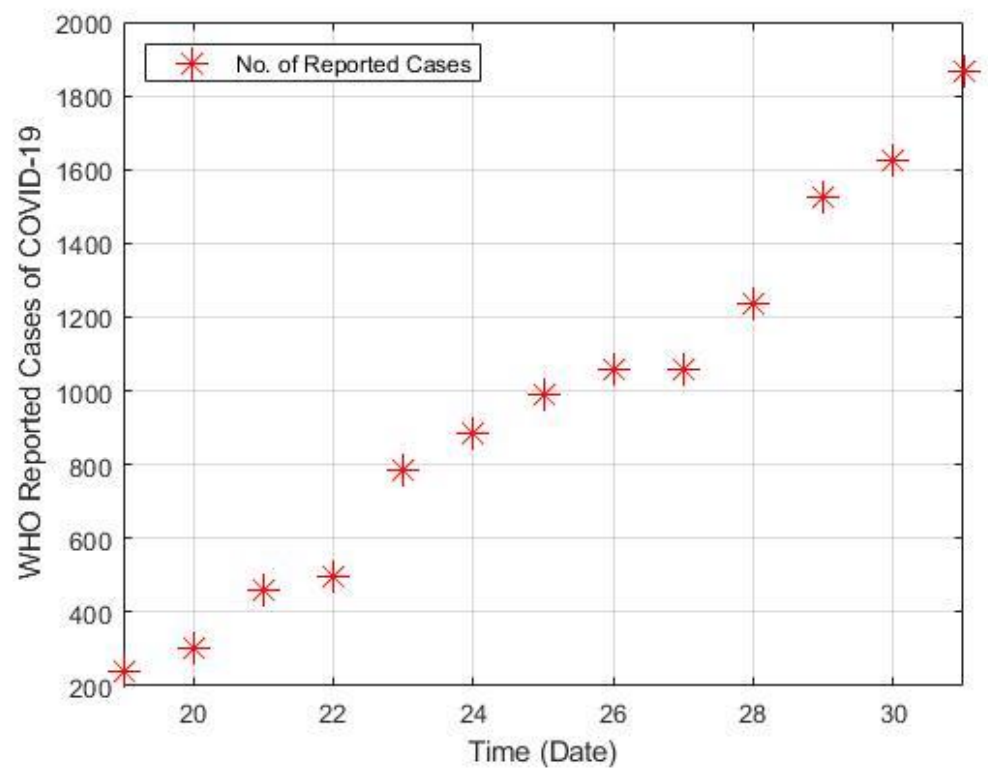

Figure 6. WHO reported cases for COVID-19 from $19^{\text {th }}$ March 2020 till $31^{\text {st }}$ March 2020.

\section{Data Fitting and Numerical Results:}

\subsection{Data Fitting:}

For data fitting of model (25) we have taken some values of parameters from the literature and the remaining values are fitted for the data collected for Pakistan. We have fitted our model solutions with the real data collected from WHO for Pakistan from $19^{\text {th }}$ March 2020 to $31^{\text {st }}$ March 2020 [5]. According to the publically reported data, the total population of Pakistan for the year 2020 is 220,410,553 [22]. For the initial values we have considered $N(0)=220,410,553$ in which the infected individuals are $I_{h}(0)=2039$ and the exposed individuals are $E_{h}(0)=10,000$. We have combined the recovered and removed population in only one class and denoted by $R_{h}(\tau)$ so that $R_{h}(0)=$ dead+recovered $=27+99=126$. The remaining population is considered as susceptible individuals so that $S_{h}(0)=220,398,388$. 


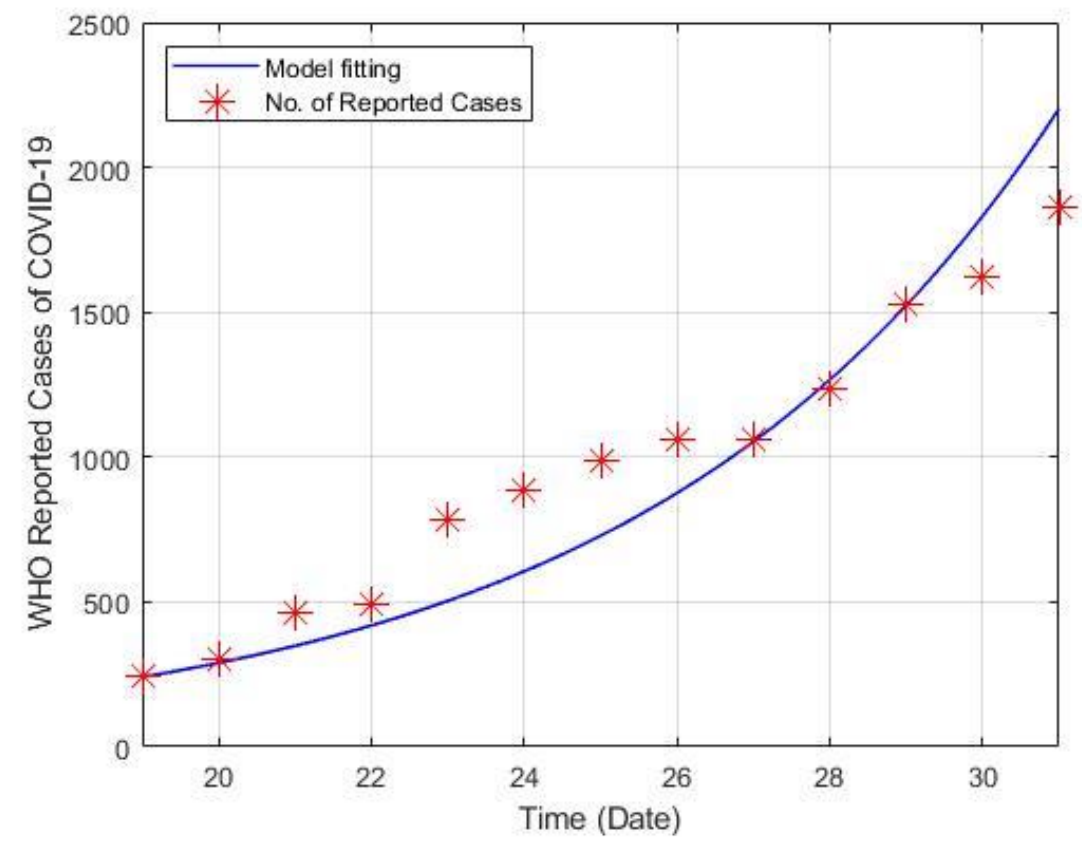

Figure 7. Real data verses model fitting

Table 1. Estimated and fitted values for different parameters of model 1.

\begin{tabular}{|c|c|c|c|}
\hline Parameter & Description & Value & Source \\
\hline$\Lambda_{h}$ & Birth rate & 3270186.2436 & Estimated \\
\hline$\eta_{h}$ & Natural Mortality rate & $\frac{1}{67.4}$ & {$[23]$} \\
& & 0.29 & Fitted \\
\hline$\lambda_{h}$ & Contact rate & 0.1243 & Fitted \\
\hline$\psi_{h}$ & Incubation period & 0.09722 & Fitted \\
\hline$\gamma_{h}$ & Recovery rate & \\
\hline
\end{tabular}

In Pakistan, for the year 2020 the life expectancy is 67.4 so that $\eta_{h}=\frac{1}{67.4}$ [23]. The corresponding birth rate in Pakistan is estimated as $\Lambda_{h}=N(0) \times \eta_{h}$ which becomes $\Lambda_{h}=3270186.2462$. Incorporating these values and after model simulation, we get different parameter values as shown in table 1. The given model is fitted for $\alpha=1$. We have estimated the basic reproduction number $\mathfrak{R}_{0} \approx 2.30748$ using different parameter values given table 1 .

\subsection{Numerical Results:}

To obtained the graphical results from the given model we have simulated the given iterative scheme through MATLAB software. Time unit is considered in days for the present 

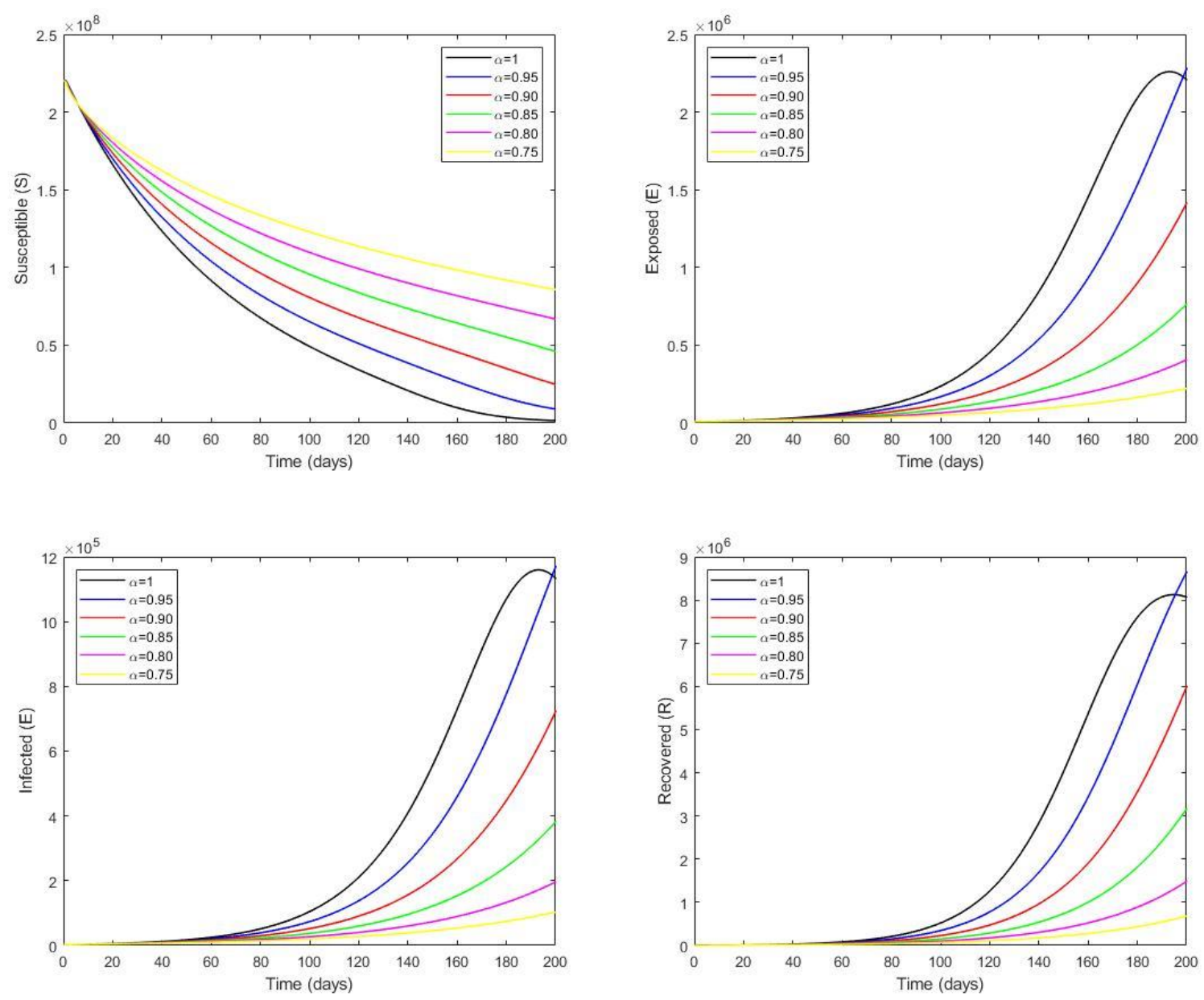

Figure 8. The dynamics of COVID-19 in different classes with different values of fractional parameter $\alpha$.

model of COVID-19. Figure 8 displays the behavior of different values of fractional parameter $\alpha$ on the dynamic and transmission of COVID-19 for different subclasses of the total population. By varying $\alpha$ we obtained variety of solutions of the given model. Furthermore, it can be observed from the figure that we have predicted the dynamics and transmission of COVID-19 for the next 200 days. Finally, figure 9 is obtained by considering the different initial values for global asymptotical stability of endemic equilibrium.

\section{Concluding Remarks:}

In the present article, we have taken SEIR model for the dynamics and transmission of COVID-19. We have also proved that the given model is bounded and invariant. Two equilibrium points DFE and EE is calculated for the steady state of the presented model. Furthermore, the basic reproduction number $\mathfrak{R}_{0}$ is calculated using next generation technique. 

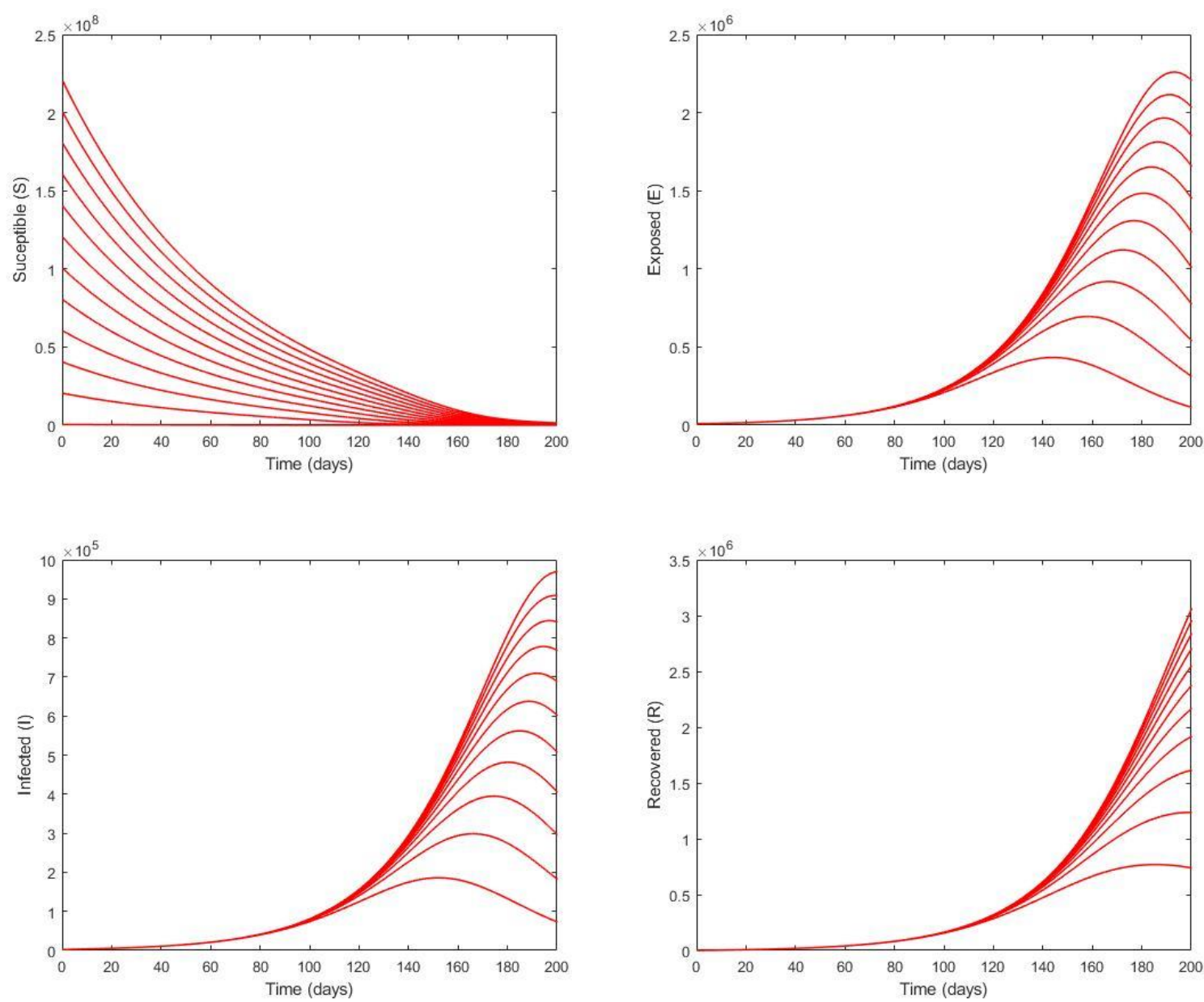

Figure 9. The dynamics of COVID-19 in different classes with different initial values.

Through stability analysis, we have concluded that the given model is locally asymptotically stable when $\Re_{0}<1$. It means that by taking $\mathfrak{R}_{0}<1$ the total population tends to DFE and the disease will die out from the population. Additionally, we have generalized the classical SEIR model by applying the recently developed AB fractional model. The real data have been fitted for the classical model i.e, $\alpha=1$. For the fitted values given in table 1 , we have approximated the basic reproduction number $\mathfrak{R}_{0} \approx 2.30748$. A numerical scheme is adopted for the solutions of $\mathrm{AB}$ fractional model. After simulation of numerical scheme different graphical results have been obtained. We have considered the unit of time in days. Furthermore, the dynamics and transmission of COVID-19 is predicted for the next 200 days. A decrease is noticed in the infected class by decreasing the values of $\mathrm{AB}$ fractional parameter $\alpha$. As the infectious diseases in not yet control in Pakistan as well as wide. We all know that the researchers and scientist are trying to find proper medical treatment or vaccination to control the spread of COVID-19. In future one may add the quarantine or vaccination or both the classes to the given model and show the effect of these classes on the spread of this deadly infectious disease. 


\section{References:}

[1]. Anderson, P. K., Cunningham, A. A., Patel, N. G., Morales, F. J., Epstein, P. R., \& Daszak, P. (2004). Emerging infectious diseases of plants: pathogen pollution, climate change and agrotechnology drivers. Trends in ecology \& evolution, 19(10), 535-544.

[2]. Wang, D., Hu, B., Hu, C., Zhu, F., Liu, X., Zhang, J., ... \& Zhao, Y. (2020). Clinical characteristics of 138 hospitalized patients with 2019 novel coronavirus-infected pneumonia in Wuhan, China. Jama, 323(11), 1061-1069.

[3]. Sanchez, Y. G., Sabir, Z., \& Guirao, J. L. (2020). Design of a nonlinear SITR fractal model based on the dynamics of a novel coronavirus (COVID.

[4]. Namendys-Silva, S. A. (2020). Respiratory support for patients with COVID-19 infection. The Lancet Respiratory Medicine, 8(4), e18.

[5]. World Health Organization. (2020). Coronavirus disease 2019 (COVID-19): situation report, 73 .

[6]. Evirgen, F., Uçar, S., \& Özdemir, N. (2020). System analysis of HIV infection model with CD4+ T under non-singular kernel derivative. Applied Mathematics and Nonlinear Sciences, 5(1), 139-146.

[7]. Qureshi, S., \& Yusuf, A. (2019). Modeling chickenpox disease with fractional derivatives: From caputo to atangana-baleanu. Chaos, Solitons \& Fractals, 122, 111118.

[8]. Prakasha, D. G., Veeresha, P., \& Baskonus, H. M. (2019). Analysis of the dynamics of hepatitis E virus using the Atangana-Baleanu fractional derivative. The European Physical Journal Plus, 134(5), 241.

[9]. Qureshi, S., \& Atangana, A. (2019). Mathematical analysis of dengue fever outbreak by novel fractional operators with field data. Physica A: Statistical Mechanics and its Applications, 526, 121127.

[10]. Koca, I. (2017). Analysis of rubella disease model with non-local and non-singular fractional derivatives. An International Journal of Optimization and Control: Theories \& Applications (IJOCTA), 8(1), 17-25.

[11]. Qureshi, S. (2020). Monotonically decreasing behavior of measles epidemic well captured by Atangana-Baleanu-Caputo fractional operator under real measles data of Pakistan. Chaos, Solitons \& Fractals, 131, 109478. 
[12]. Sweilam, N. H., Al-Mekhlafi, S. M., \& Baleanu, D. (2019). Optimal control for a fractional tuberculosis infection model including the impact of diabetes and resistant strains. Journal of advanced research, 17, 125-137.

[13]. Arif, M., Ali, F., Sheikh, N. A., Khan, I., \& Nisar, K. S. (2019). Fractional Model of Couple Stress Fluid for Generalized Couette Flow: A Comparative Analysis of Atangana-Baleanu and Caputo-Fabrizio Fractional Derivatives. IEEE Access, 7, 88643-88655.

[14]. Arif, M., Ali, F., Khan, I., \& Nisar, K. S. (2020). A Time Fractional Model With NonSingular Kernal the Generalized Couette Flow of Couple Stress Nanofluid. IEEE Access.

[15]. Sheikh, N. A., Ali, F., Khan, I., Gohar, M., \& Saqib, M. (2017). On the applications of nanofluids to enhance the performance of solar collectors: A comparative analysis of Atangana-Baleanu and Caputo-Fabrizio fractional models. The European Physical Journal Plus, 132(12), 540.

[16]. Doungmo Goufo, E. F. (2016). Chaotic processes using the two-parameter derivative with non-singular and non-local kernel: Basic theory and applications. Chaos: An Interdisciplinary Journal of Nonlinear Science, 26(8), 084305.

[17]. Gómez-Aguilar, J. F., Atangana, A., \& Morales-Delgado, V. F. (2017). Electrical circuits RC, LC, and RL described by Atangana-Baleanu fractional derivatives. International Journal of Circuit Theory and Applications, 45(11), 15141533.

[18]. Van den Driessche, P., \& Watmough, J. (2002). Reproduction numbers and subthreshold endemic equilibria for compartmental models of disease transmission. Mathematical biosciences, 180(1-2), 29-48.

[19]. A. Atangana, 'On the new fractional derivative and application to nonlinear Fisher's reaction-diffusion equation,'” Appl. Math. Comput., vol. 273, pp. 948-956, Jan. 2016.

[20]. Sene, N. (2020). SIR epidemic model with Mittag-Leffler fractional derivative. Chaos, Solitons \& Fractals, 137, 109833.

[21]. Toufik, M., \& Atangana, A. (2017). New numerical approximation of fractional derivative with non-local and non-singular kernel: Application to chaotic models. The European Physical Journal Plus, 132(10), 444.

[22]. Population of Pakistan, https://www.worldometers.info/world-population/pakistanpopulation

[23]. Life expectancy in Pakistan, https://www.worldlifeexpectancy.com/pakistan-lifeexpectancy

\section{Declarations:}

The authors declare no competing interest. 
Figures

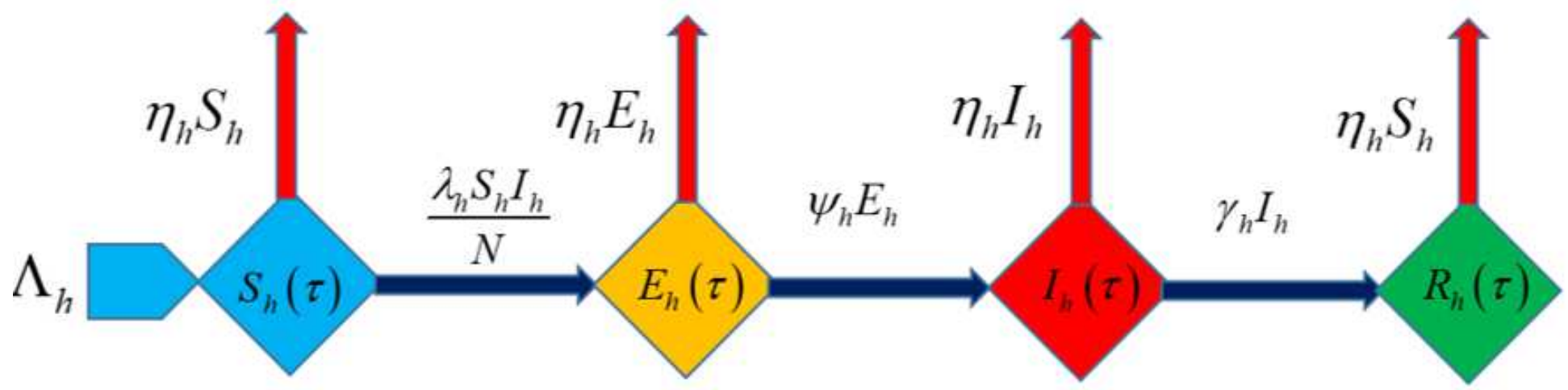

Figure 1

Flow chart of the given SEIR model

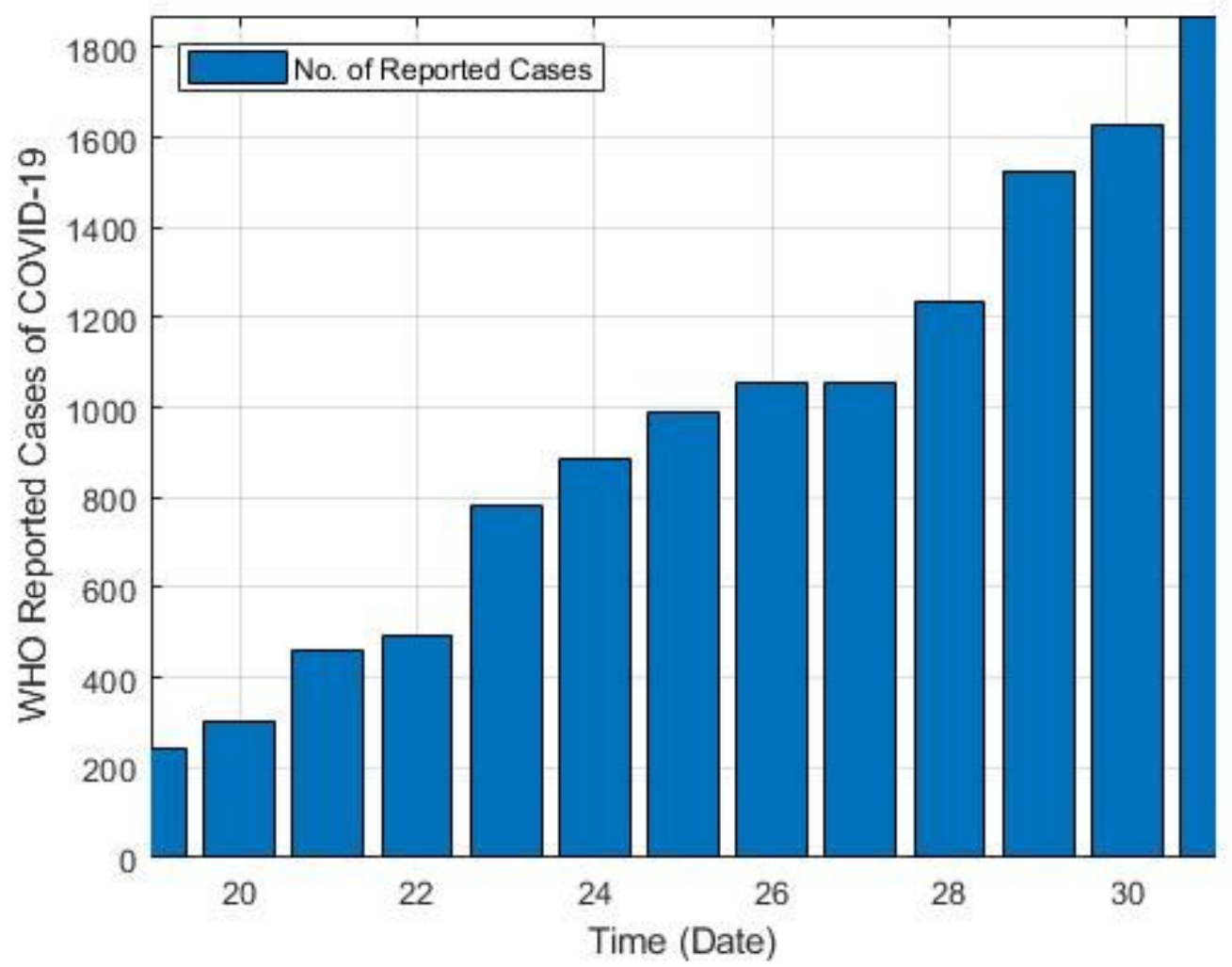

Figure 2

WHO reported cases for COVID-19 from 19th March 2020 till 31st March 2020 


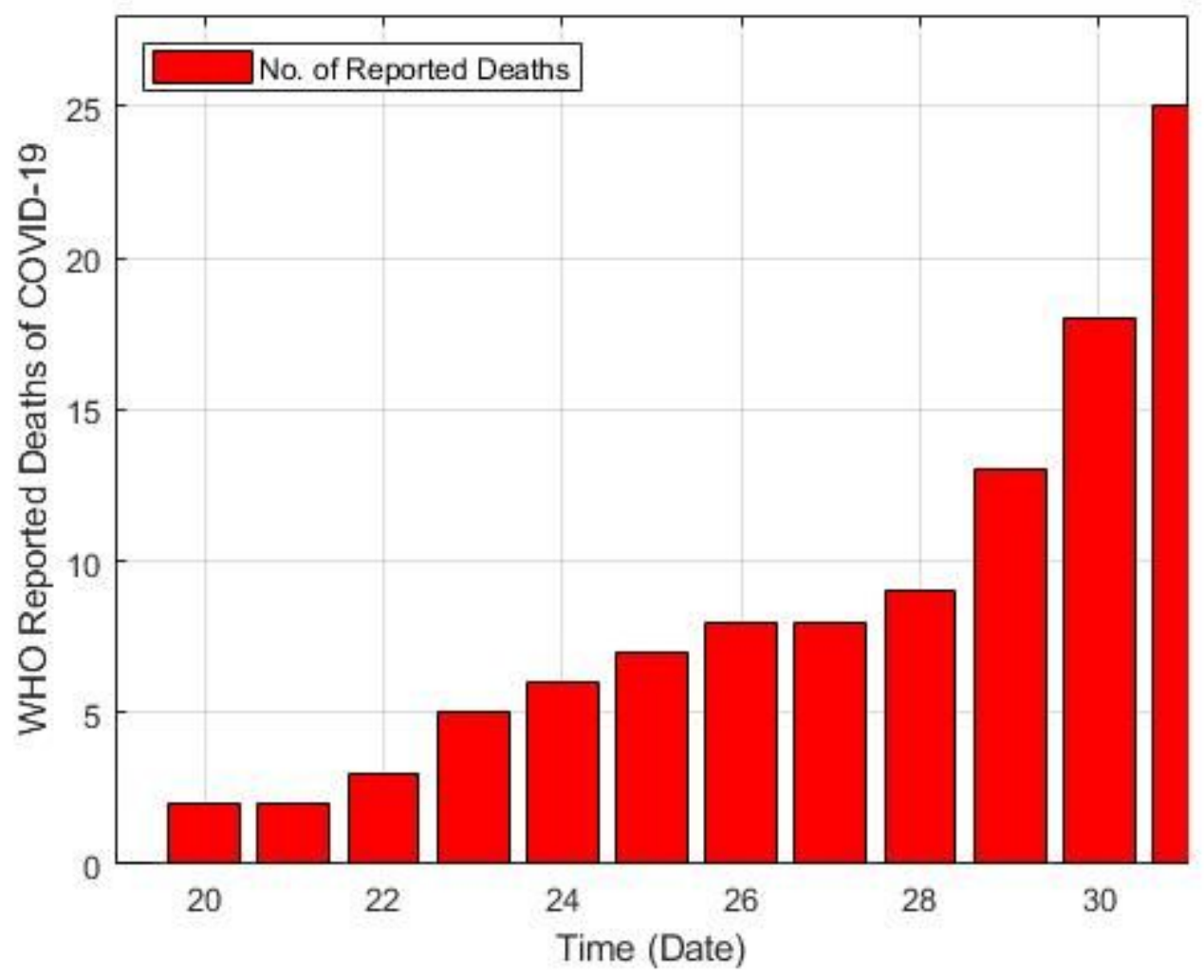

Figure 3

WHO reported deaths by COVID-19 from 19th March 2020 till 31st March 2020

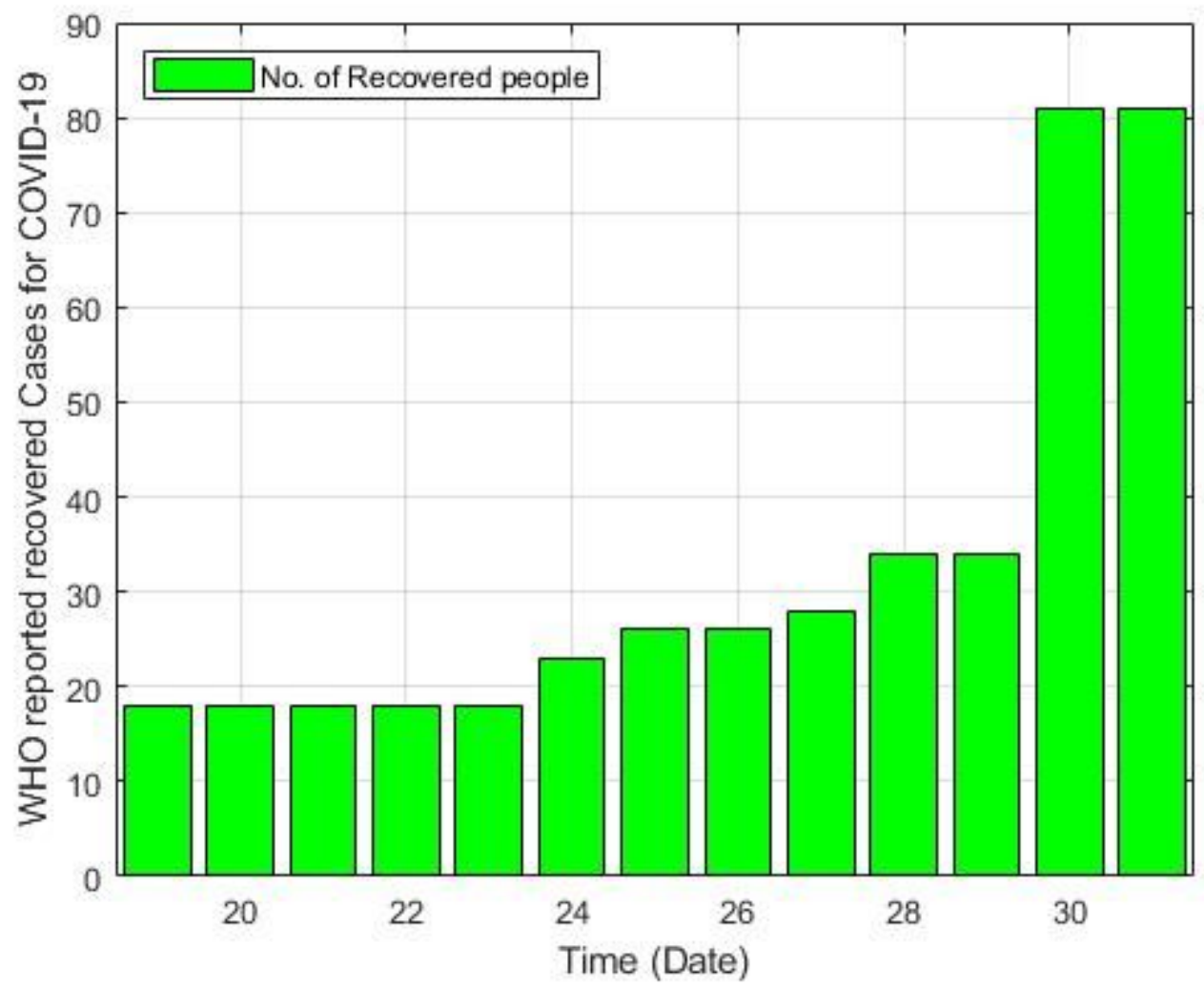


Figure 4

WHO reported deaths by COVID-19 from 19th March 2020 till 31st March 2020

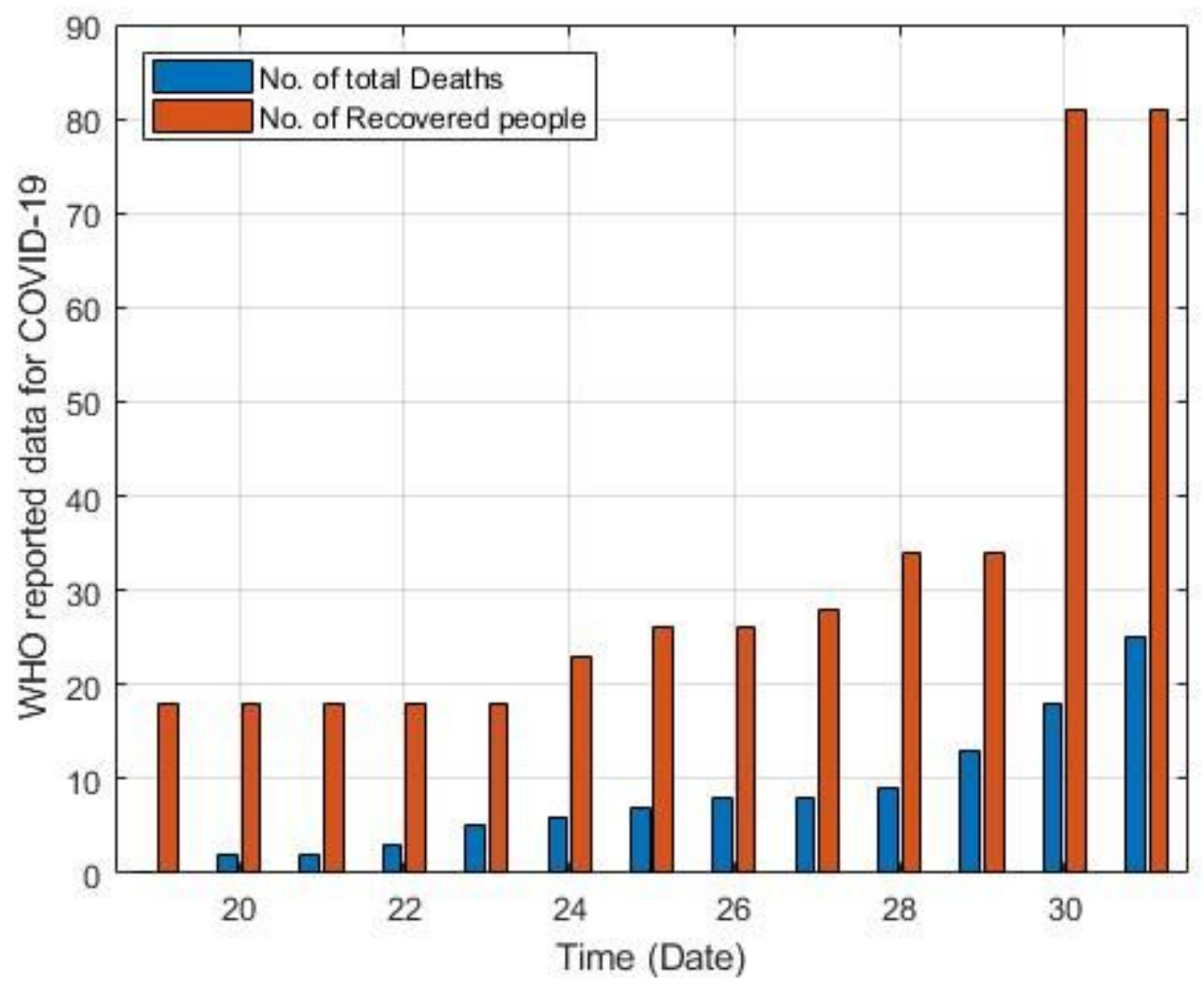

Figure 5

Comparative bar chart of reported deaths and recovered individuals of COVID-19 


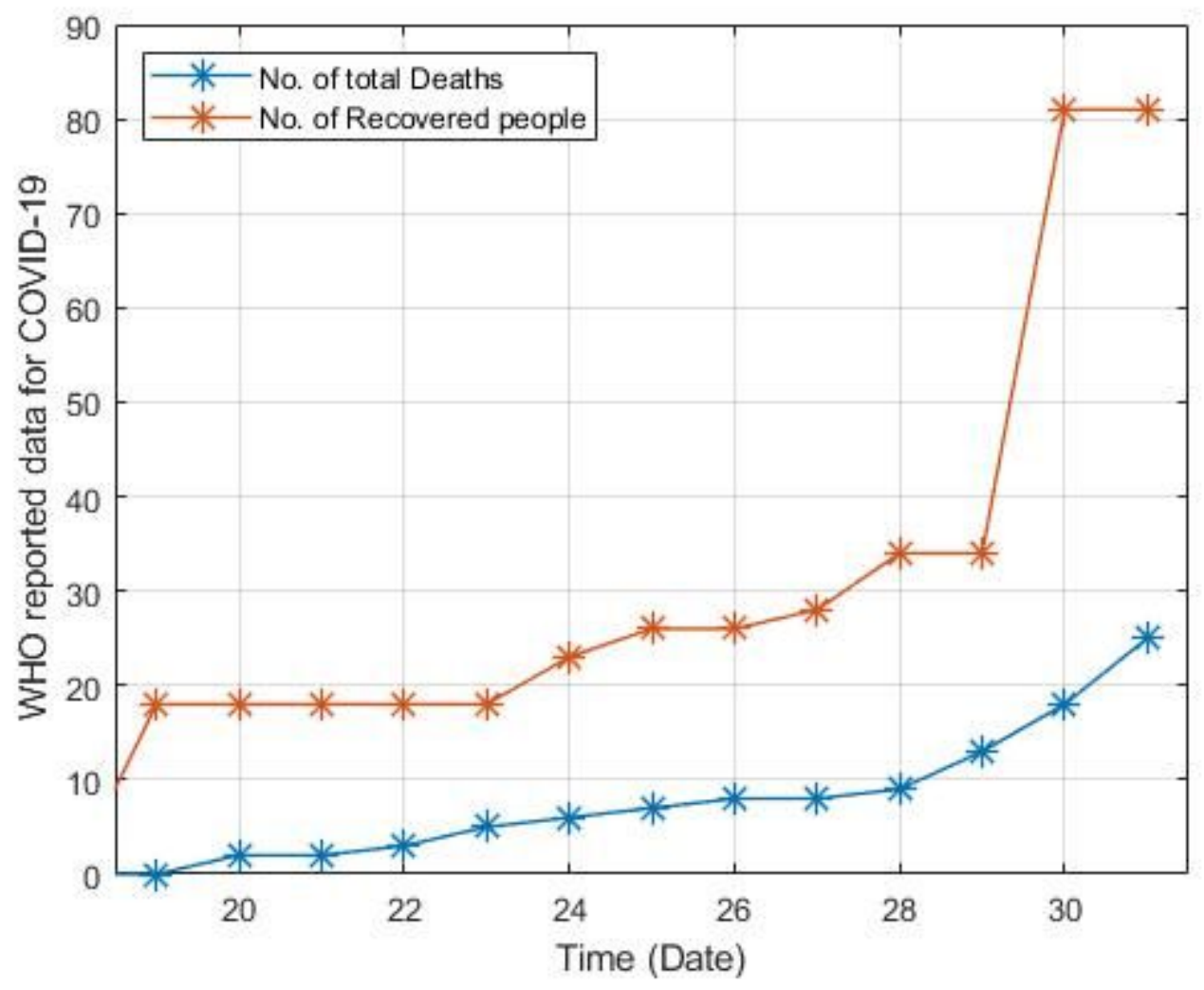

Figure 6

Comparative line plot of reported deaths and recovered individuals of COVID-19

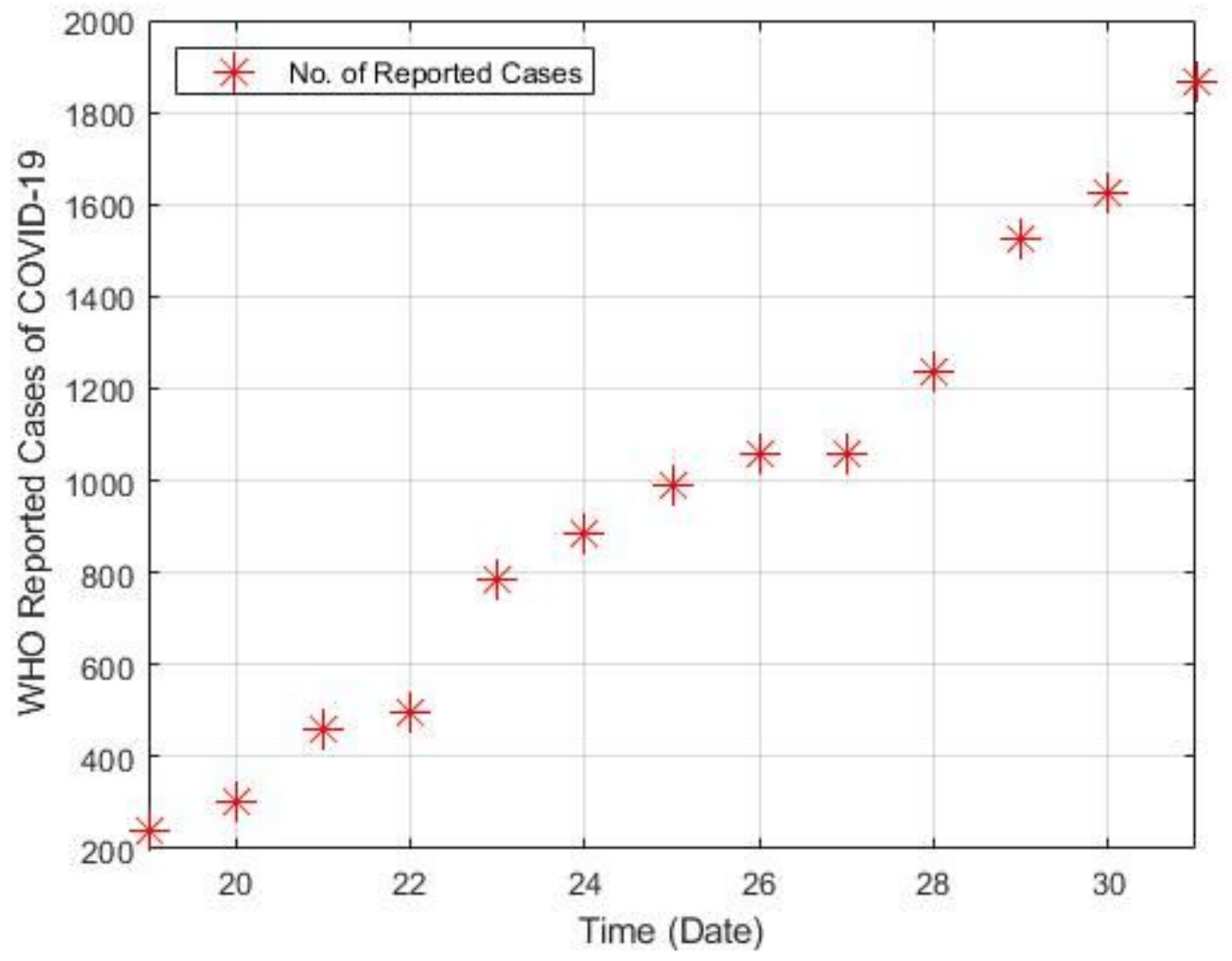


Figure 7

WHO reported cases for COVID-19 from 19th March 2020 till 31st March 2020

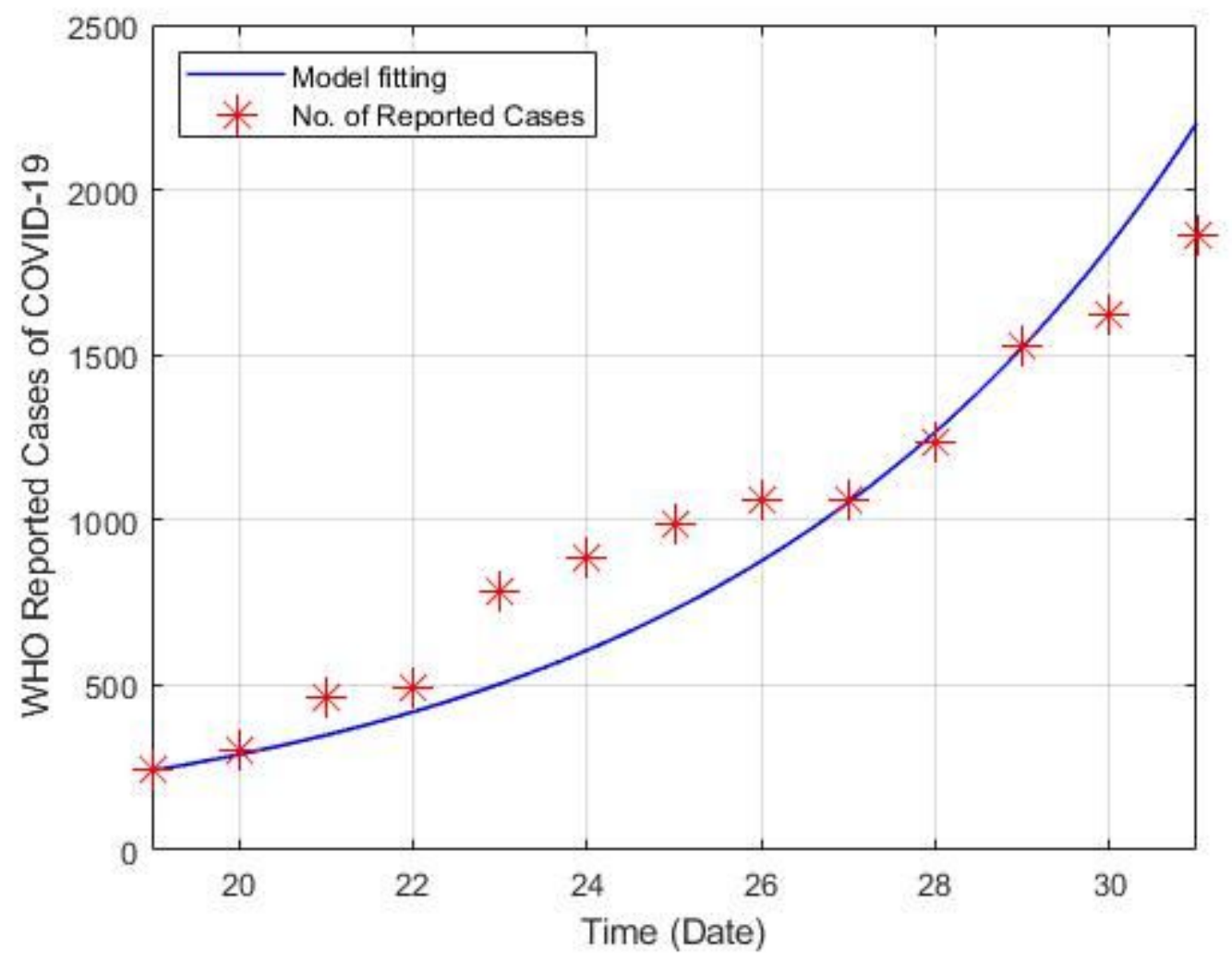

Figure 8

Real data versus model fitting 


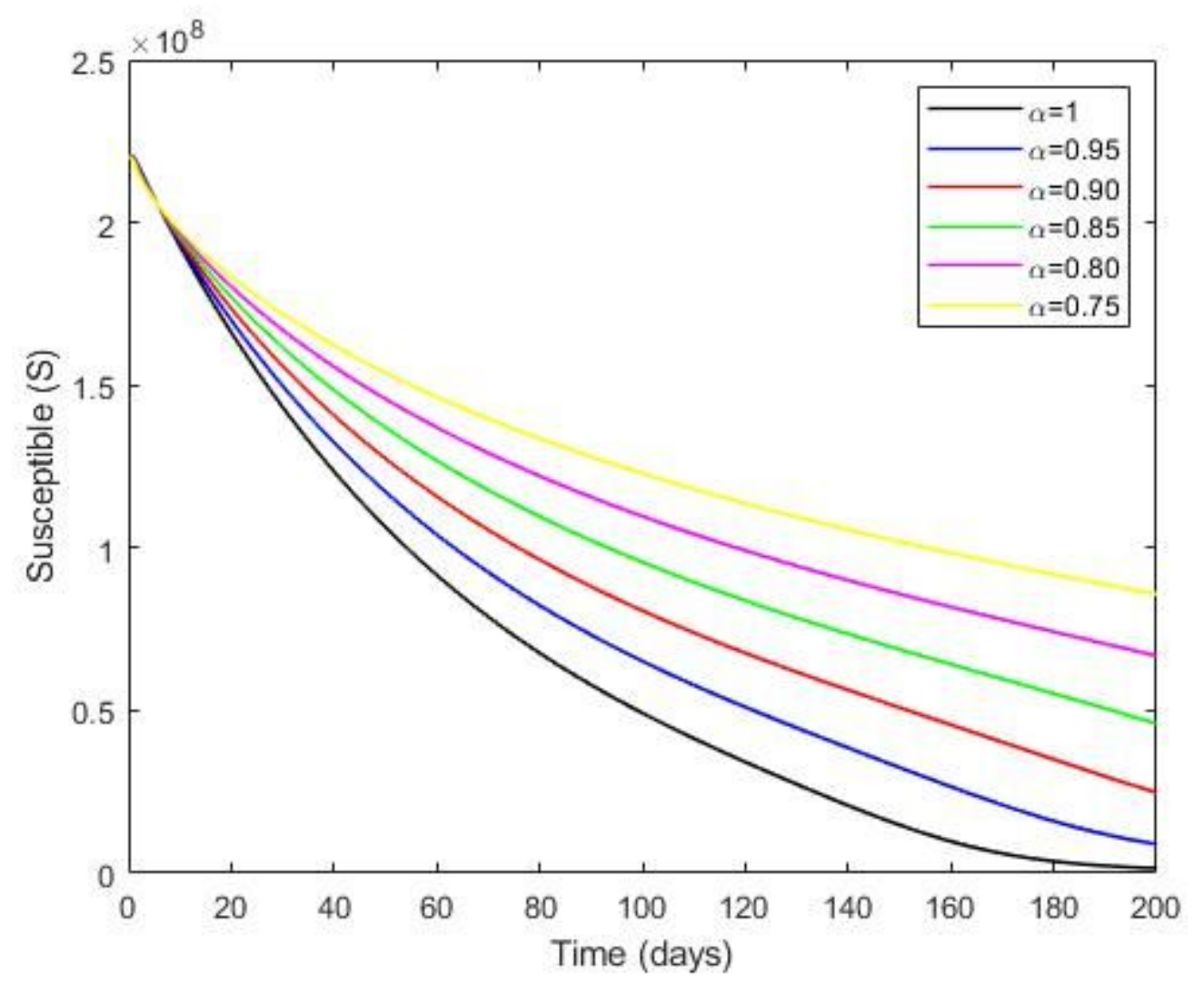

Figure 9

The dynamics of COVID-19 in Susceptible class with different values of fractional parameter .

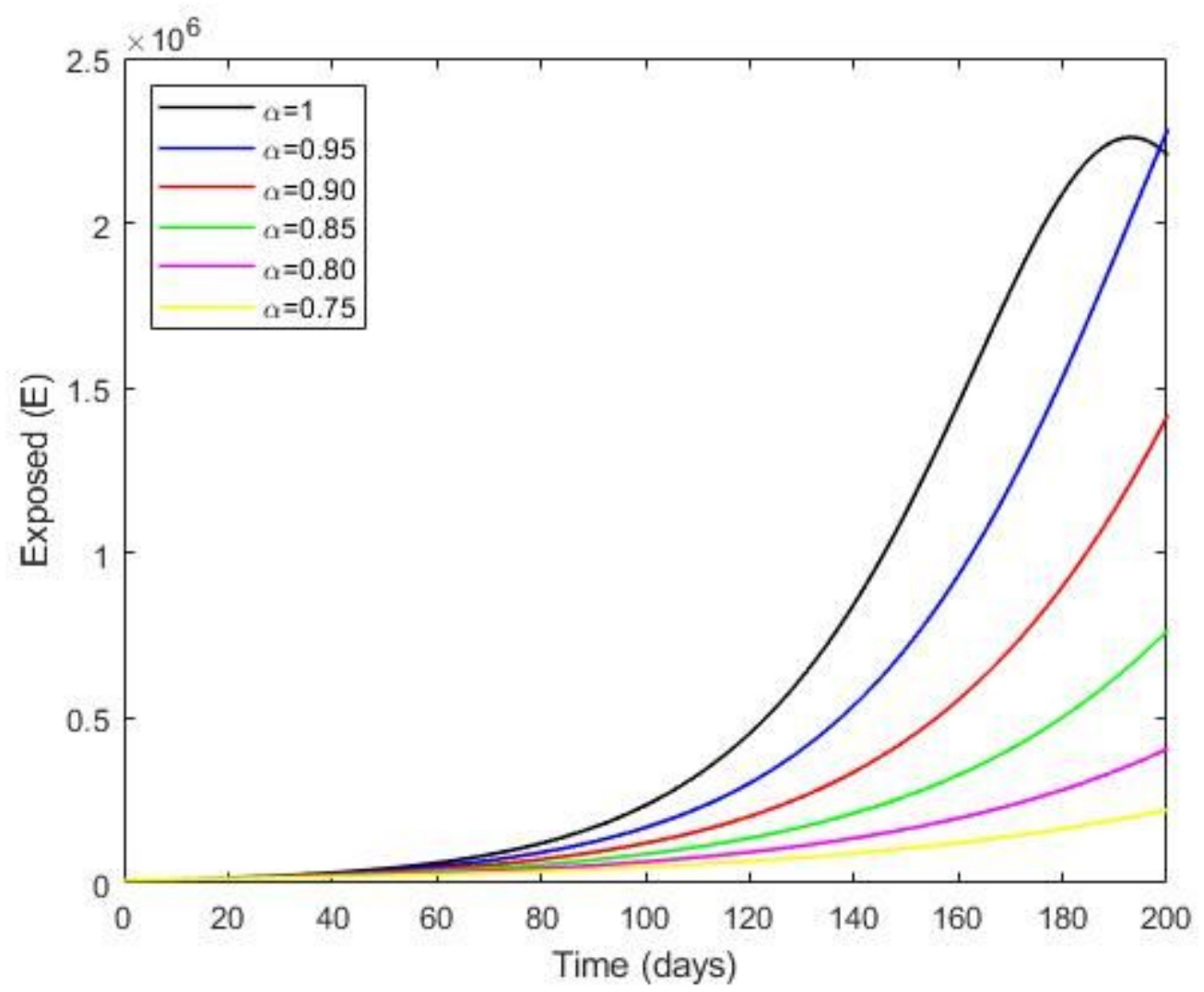


Figure 10

The dynamics of COVID-19 in exposed class with different values of fractional parameter .

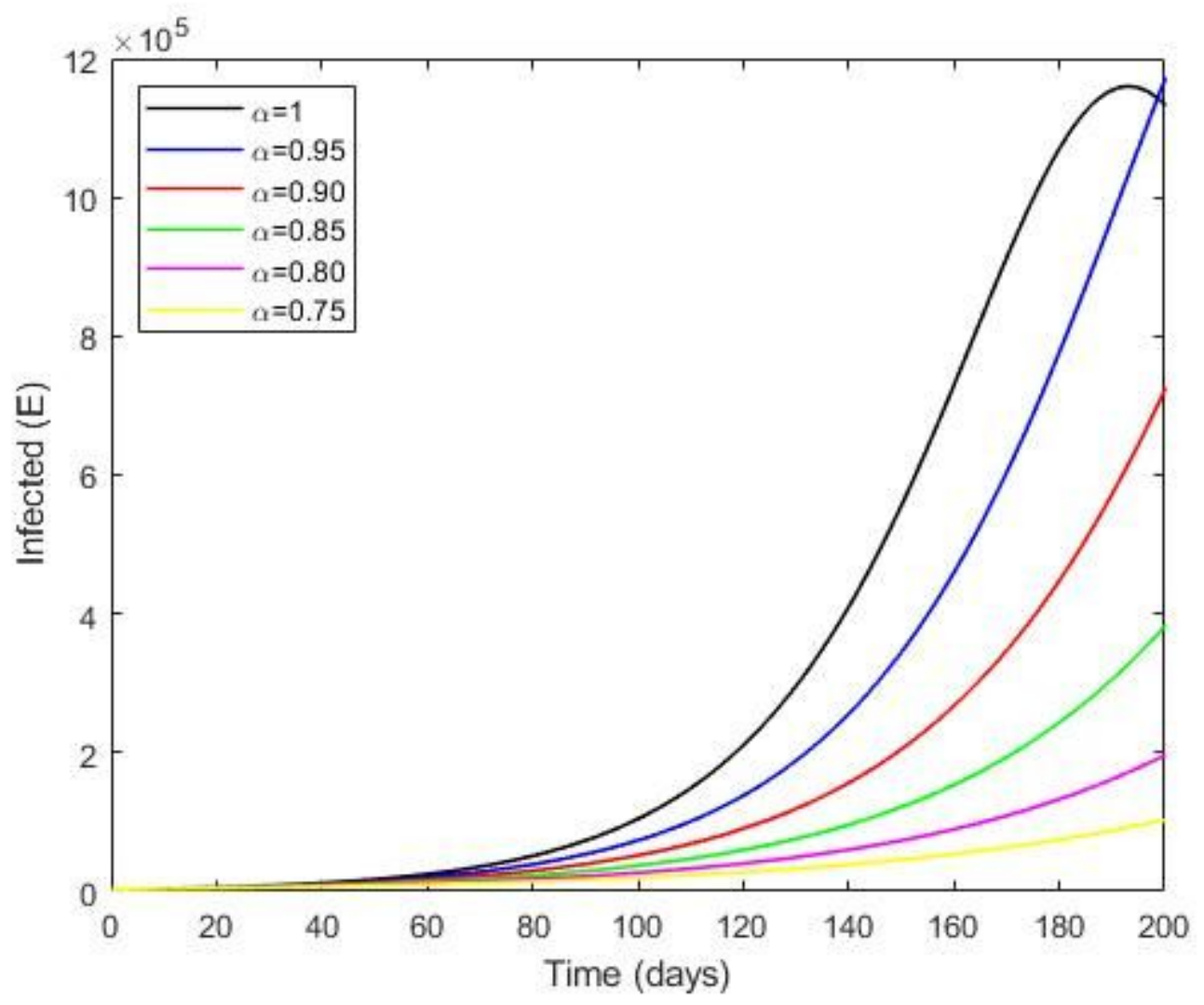

Figure 11

The dynamics of COVID-19 in infected class with different values of fractional parameter . 


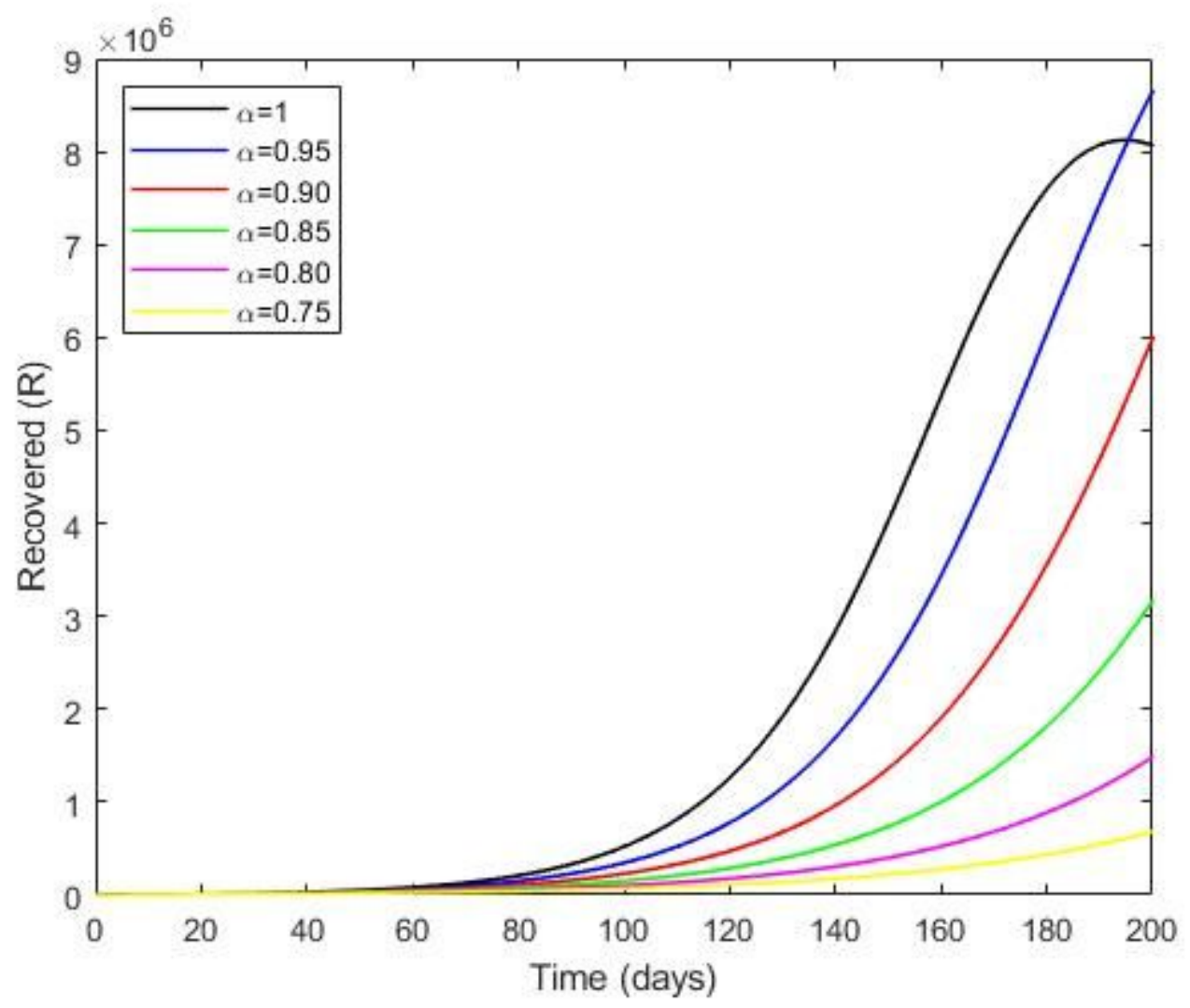

Figure 12

The dynamics of COVID-19 in recovered class with different values of fractional parameter .

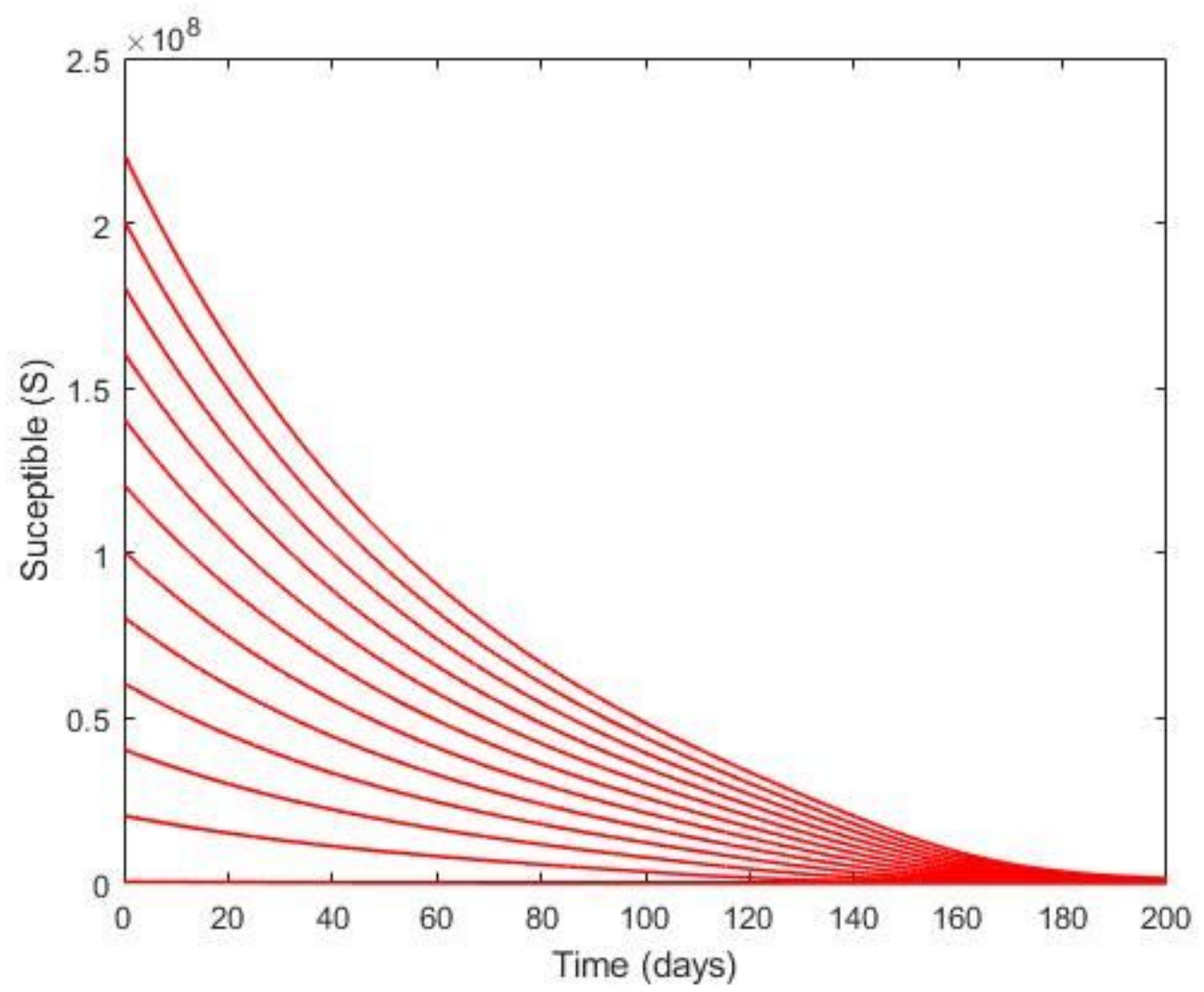


Figure 13

The dynamics of COVID-19 in susceptible class with different initial values.

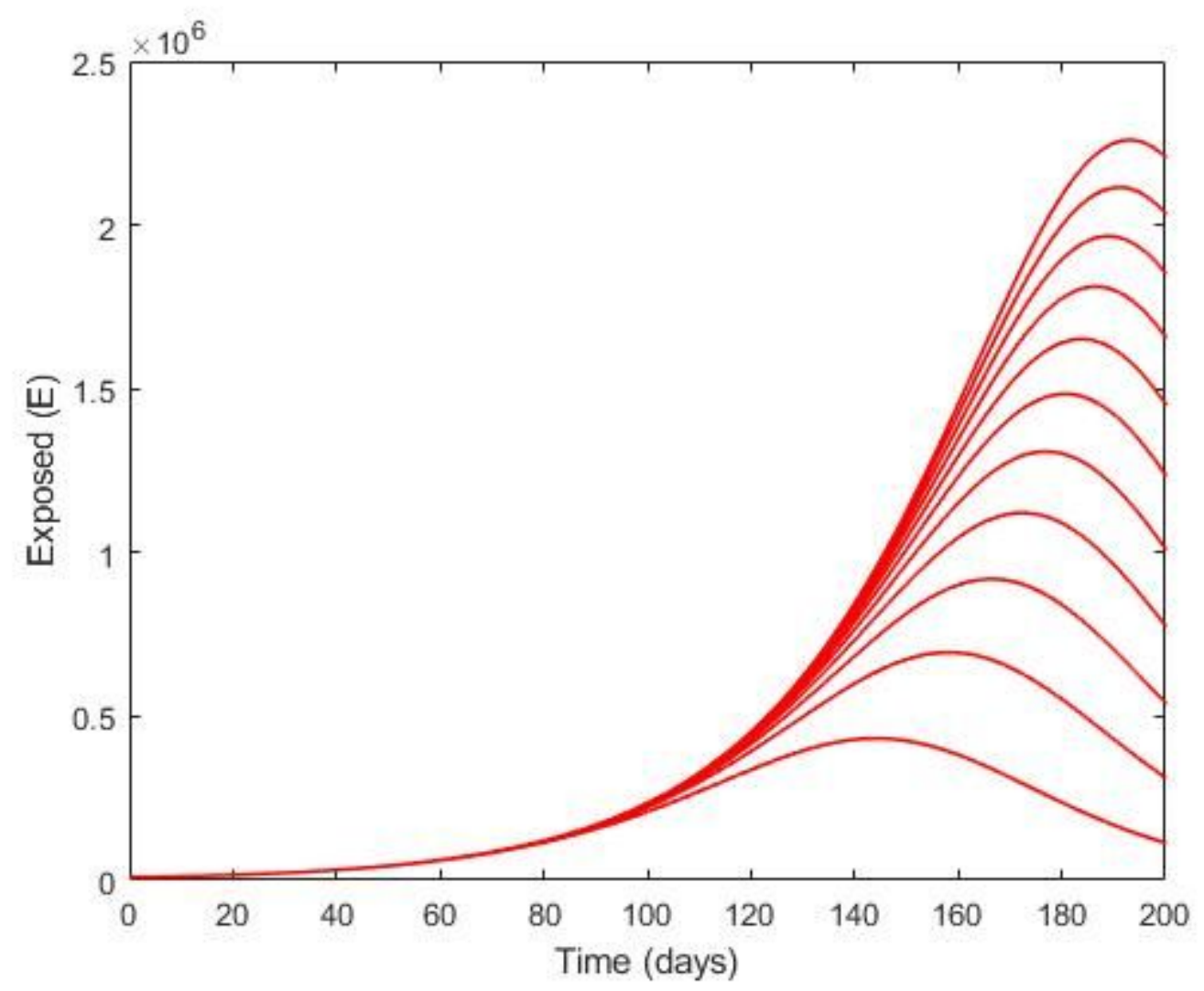

Figure 14

The dynamics of COVID-19 in exposed class with different initial values. 


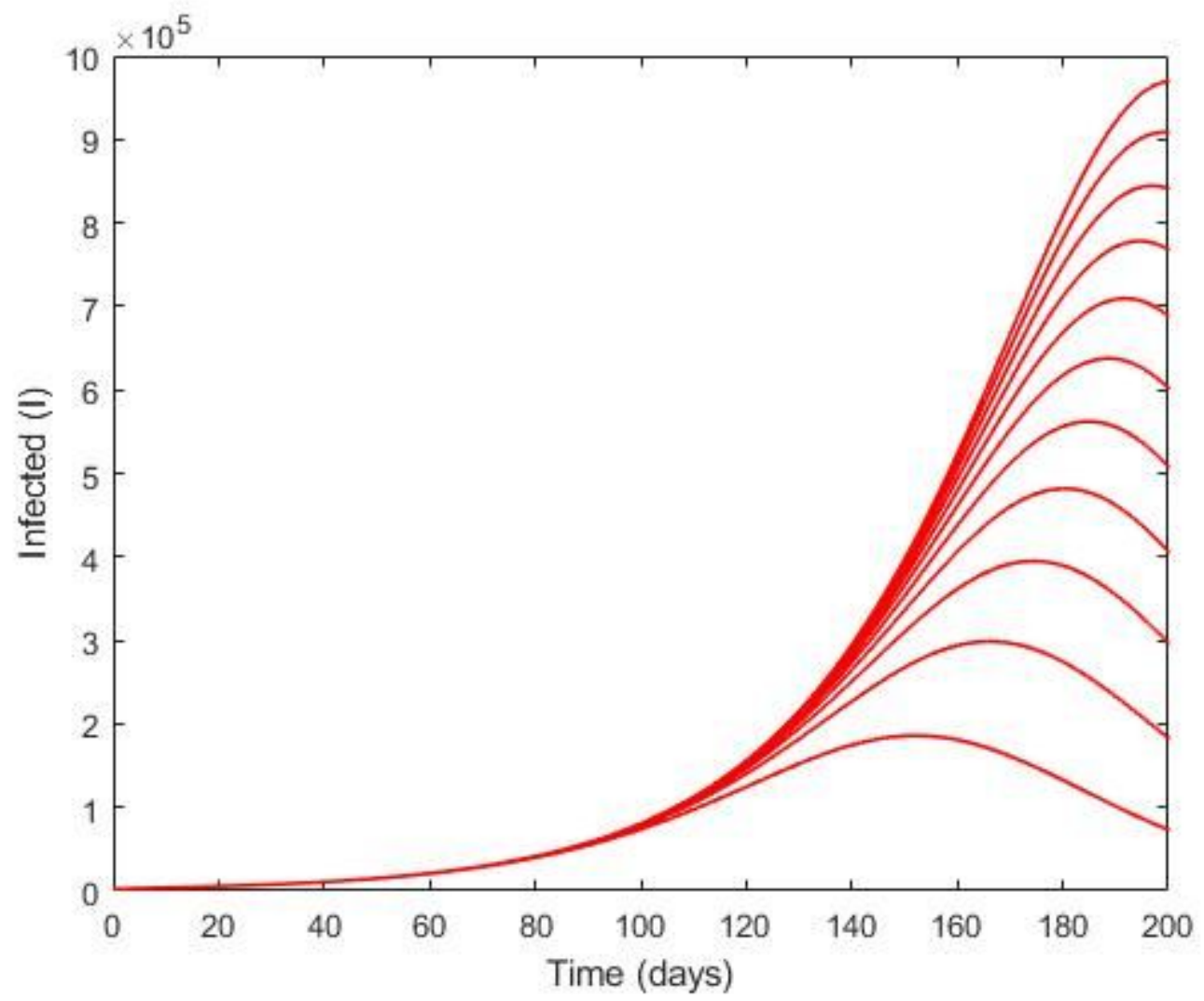

\section{Figure 15}

The dynamics of COVID-19 in infected class with different initial values.

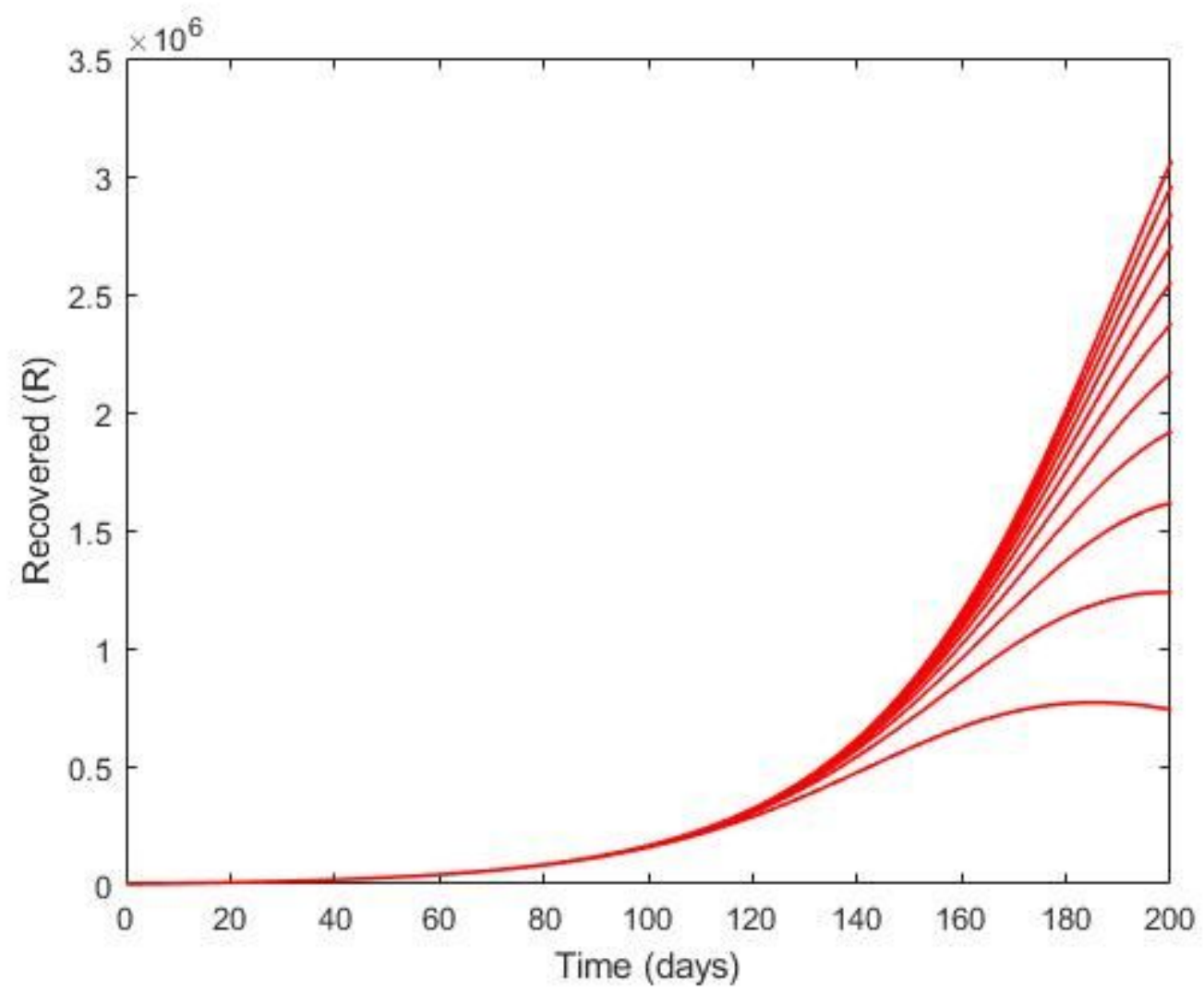


Figure 16

The dynamics of COVID-19 in recovered class with different initial values. 